\title{
The Muskat problem with surface tension and equal viscosities in subcritical $L_{p}$-Sobolev spaces
}

\author{
Anca-Voichita Matioc ${ }^{1} \cdot$ Bogdan-Vasile Matioc $^{1}$ (D)
}

Received: 29 October 2020 / Accepted: 10 June 2021 / Published online: 29 June 2021

(c) The Author(s) 2021

\section{Abstract}

In this paper we establish the well-posedness of the Muskat problem with surface tension and equal viscosities in the subcritical Sobolev spaces $W_{p}^{s}(\mathbb{R})$, where $p \in(1,2]$ and $s \in(1+1 / p, 2)$. This is achieved by showing that the mathematical model can be formulated as a quasilinear parabolic evolution problem in $W_{p}^{\bar{s}-2}(\mathbb{R})$, where $\bar{s} \in(1+1 / p, s)$. Moreover, we prove that the solutions become instantly smooth and we provide a criterion for the global existence of solutions.

Keywords Muskat problem · Quasilinear parabolic evolution equation · Surface tension · Singular integral

Mathematics Subject Classification 35R37 · 76D27 · 35K59

\section{Introduction}

In this paper we study the evolution equation

$$
\partial_{t} f(t, x)=\frac{k}{2 \pi \mu} \mathrm{PV} \int_{\mathbb{R}} \frac{y+\partial_{x} f(t, x) \delta_{[x, y]} f(t)}{y^{2}+\left(\delta_{[x, y]} f(t)\right)^{2}} \partial_{x}\left(\sigma \kappa(f)-\Delta_{\rho} f\right)(t, x-y) d y,
$$

which is defined for $t>0$ and $x \in \mathbb{R}$. The function $f$ is assumed to be known at time $t=0$, that is

$$
f(0, \cdot)=f_{0}
$$

Bogdan-Vasile Matioc bogdan.matioc@ur.de

Anca-Voichita Matioc anca.matioc@ur.de

1 Fakultät für Mathematik, Universität Regensburg, 93040 Regensburg, Germany 
The evolution problem (1a) is the contour integral formulation of the Muskat problem with surface tension and with/without gravity effects, see [24, 25] for an equivalence proof of (1a) to the classical formulation of the Muskat problem [29]. The problem (1a) describes the two-dimensional motion of two fluids with equal viscosities $\mu_{-}=\mu_{+}=\mu$ and general densities $\rho_{-}$and $\rho_{+}$in a vertical/horizontal homogeneous porous medium which is identified with $\mathbb{R}^{2}$. The fluids occupy the entire plane, they are separated by the sharp interface $\{y=f(t, x)+V t\}$, and they move with constant velocity $(0, V)$, where $V \in \mathbb{R}$. The fluid denoted by + is located above this moving interface. We use $g \in[0, \infty)$ to denote the Earth's gravity, $k>0$ is the permeability of the homogeneous porous medium, and $\sigma>0$ is the surface tension coefficient at the free boundary. Moreover, to shorten the notation we have set $\Delta_{\rho}:=g\left(\rho_{-}-\rho_{+}\right) \in \mathbb{R}$ and

$$
\delta_{[x, y]} f:=f(x)-f(x-y), \quad x, y \in \mathbb{R} .
$$

Finally, $\kappa(f(t))$ is the curvature of $\{y=f(t, x)+t V\}$ and PV denotes the principal value.

The Muskat problem with surface tension has received much interest in the recent years. Besides the fundamental well-posedness issue also other important features like the stability of stationary solutions [13, 14, 16, 20, 26, 28, 33, 36], parabolic smoothing properties [24-26], the zero surface tension limit [8, 31], and the degenerate limit when the thickness of the fluid layers (or a certain nondimensional parameter) vanishes $[15,22,37]$ have been investigated in this context. We also refer to $[19,21]$ for results on the Hele-Shaw problem with surface tension effects, which is the one-phase version of the Muskat problem, and to [34, $35,38]$ for results on the related Verigin problem with surface tension.

Concerning the well-posedness of the Muskat problem with surface tension effects, this property has been investigated in bounded (layered) geometries in $[14,16,17,33,36]$ where abstract parabolic theories have been employed in the analysis, the approach in [23] relies on Schauder's fixed-point theorem, and in [10] the authors use Schauder's fixed-point theorem in a setting which allows for a sharp corner point of the initial geometry.

The results on the Muskat problem with surface tension in the unbounded geometry considered in this paper (and possibly in the general case of fluids with different viscosities) are more recent, cf. [8, 24-26, 30, 31, 39]. While in [8, 39] the initial data are chosen from $H^{s}(\mathbb{T})$, with $s \geq 6$, the regularity of the initial data has been decreased in [24-26] to $H^{2+\varepsilon}(\mathbb{R})$, with $\varepsilon \in(0,1)$ arbitrarily small. Finally, the very recent references $[30,31]$ consider the problem with initial data in $H^{1+\frac{d}{2}+\varepsilon}\left(\mathbb{R}^{d}\right)$, with $d \geq 1$ and $\varepsilon>0$ arbitrarily small, covering all subcritical $L_{2}$ -based Sobolev spaces in all dimensions.

It is the aim of this paper to study the Muskat problem (1a) in the subcritical $L_{p}$-based Sobolev spaces $W_{p}^{s}(\mathbb{R})$ with $p \in(1,2]$ and $s \in(1+1 / p, 2)$. This issue is new in the context of (1a) ( see $[2,12]$ for results in the case when $\sigma=0$ ). To motivate why $W_{p}^{1+1 / p}(\mathbb{R})$ is a critical space for (1a) we first emphasize that the surface tension is the dominant factor for the dynamics as it contains the highest 
spatial derivatives of $f$. Besides, if we set $g=0$, then it is not difficult to show that if $f$ is a solution to (1a), then, given $\lambda>0$, the function

$$
f_{\lambda}(t, x):=\lambda^{-1} f\left(\lambda^{3} t, \lambda x\right)
$$

also solves (1a). This scaling identifies $W_{p}^{1+1 / p}(\mathbb{R})$ as a critical space for (1a). The main result Theorem 1.1 establishes the well-posedness of $(1 \mathrm{a})$ in $W_{p}^{s}(\mathbb{R})$. This is achieved by showing that (1a) can be recast as a quasilinear parabolic evolution equation, so that abstract results available for this type of problems, cf. [3, 4, 6, 28], can be applied in our context. A particular feature of the Muskat problem (1a) is the fact that the equations have to be interpreted in distributional sense as they are realized in the Sobolev space $W_{p}^{\bar{s}-2}(\mathbb{R})$, where $\bar{s}$ is chosen such that $1+1 / p<\bar{s}<s<2$. Additionally to well-posedness, Theorem 1.1 provides two parabolic smoothing properties, showing in particular that (1a) holds pointwise, and a criterion for the global existence of solutions.

Theorem 1.1 Let $p \in(1,2]$ and $1+1 / p<\bar{s}<s<2$. Then, the Muskat problem (1a) possesses for each $f_{0} \in W_{p}^{s}(\mathbb{R})$ a unique maximal solution $f:=f\left(\cdot ; f_{0}\right)$ such that

$$
f \in \mathrm{C}\left(\left[0, T^{+}\right), W_{p}^{s}(\mathbb{R})\right) \cap \mathrm{C}\left(\left(0, T^{+}\right), W_{p}^{\bar{s}+1}(\mathbb{R})\right) \cap \mathrm{C}^{1}\left(\left(0, T^{+}\right), W_{p}^{\bar{s}-2}(\mathbb{R})\right),
$$

with $T^{+}=T^{+}\left(f_{0}\right) \in(0, \infty]$ denoting the maximal time of existence. Moreover, the following properties hold true:

(i) The solution depends continuously on the initial data;

(ii) Given $k \in \mathbb{N}$, we have $f \in \mathrm{C}^{\infty}\left(\left(0, T^{+}\right) \times \mathbb{R}, \mathbb{R}\right) \cap \mathrm{C}^{\infty}\left(\left(0, T^{+}\right), W_{p}^{k}(\mathbb{R})\right)$;

(iii) The solution is global if

$$
\sup _{\left[0, T^{+}\right) \cap[0, T]}\|f(t)\|_{W_{p}^{s}(\mathbb{R})}<\infty \quad \text { for all } \quad T>0 .
$$

We emphasize that some of the arguments use in an essential way the fact that $p \in(1,2]$. More precisely, we employ several times the Sobolev embedding $W_{p}^{s}(\mathbb{R}) \hookrightarrow W_{p^{\prime}}^{t}(\mathbb{R})$, where $p^{\prime}$ is the adjoint exponent of $p$, that is $p^{-1}+p^{\prime-1}=1$ (this notation is used in the entire paper). This provides the restriction $p \in(1,2]$.

Additionally, we expect that the assertion (ii) of Theorem 1.1 can be improved to real-analyticity instead of smoothness. However, this would require to establish real-analytic dependence of the right-hand side of (1a) on $f$ in the functional analytic framework considered in Sect. 3, which is much more involved than showing the smooth dependence (see [25, Proposition 5.1] for a related proof of real-analyticity).

\subsection{Notation}

Given $k \in \mathbb{N}$ and $p \in(1, \infty)$, we let $W_{p}^{k}(\mathbb{R})$ denote the standard $L_{p}$-based Sobolev space with norm 


$$
\|f\|_{W_{p}^{k}}:=\left(\sum_{\ell=0}^{k}\left\|f^{(\ell)}\right\|_{p}^{p}\right)^{1 / p}
$$

Given $0<s \notin \mathbb{N}$ with $s=[s]+\{s\}$, where $[s] \in \mathbb{N}$ and $\{s\} \in(0,1)$, the Sobolev space $W_{p}^{s}(\mathbb{R})$ is the subspace of $W_{p}^{[s]}(\mathbb{R})$ that consists of functions for which the seminorm

$$
[f]_{W_{p}^{s}}^{p}:=\int_{\mathbb{R}^{2}} \frac{\left|f^{([s])}(x)-f^{([s])}(y)\right|^{p}}{|x-y|^{1+\{s\} p}} d(x, y)=\int_{\mathbb{R}} \frac{\left\|f^{([s])}-\tau_{\xi} f^{([s])}\right\|_{p}^{p}}{|\xi|^{1+\{s\} p}} d \xi
$$

is finite. Here $\left\{\tau_{\xi}\right\}_{\xi \in \mathbb{R}}$ is the group of right translations and $\|\cdot\|_{q}:=\|\cdot\|_{L_{q}(\mathbb{R})}$ ,$q \in[1, \infty]$. The norm on $W_{p}^{s}(\mathbb{R})$ is defined by

$$
\|f\|_{W_{p}^{s}}:=\left(\|f\|_{W_{p}^{[s]}}^{p}+[f]_{W_{p}^{s}}^{p}\right)^{1 / p}
$$

For $s<0, W_{p}^{s}(\mathbb{R})$ is defined as the dual space of $W_{p^{\prime}}^{-s}(\mathbb{R})$.

The following properties can be found e.g. in [40].

(i) $\quad \mathrm{C}_{0}^{\infty}(\mathbb{R})$ lies dense in $W_{p}^{s}(\mathbb{R})$ for all $s \in \mathbb{R}$. Moreover, $W_{p}^{s}(\mathbb{R}) \hookrightarrow \mathrm{C}^{s-1 / p}(\mathbb{R})$ holds provided that $0<s-1 / p \notin \mathbb{N}$.

(ii) $W_{p}^{s}(\mathbb{R})$ is an algebra for $s>1 / p$.

(iii) If $\rho>\max \{s,-s\}$, then $f \in \mathrm{C}^{\rho}(\mathbb{R})$ is a pointwise multiplier for $W_{p}^{s}(\mathbb{R})$, that is

$$
\|f g\|_{W_{p}^{s}} \leq C\|f\|_{C^{\rho}}\|g\|_{W_{p}^{s}} \quad \text { for all } g \in W_{p}^{s}(\mathbb{R}),
$$

with $C$ independent of $f$ and $g$.

(iv) Given $\theta \in(0,1)$ and $p \in(1, \infty)$, let $(\cdot, \cdot)_{\theta, p}$ denote the real interpolation functor of exponent $\theta$ and parameter $p \in(1, \infty)$. Given $s_{0}, s_{1} \in \mathbb{R}$ with $(1-\theta) s_{0}+\theta s_{1} \notin \mathbb{Z}$, it holds that

$$
\left(W_{p}^{S_{0}}(\mathbb{R}), W_{p}^{s_{1}}(\mathbb{R})\right)_{\theta, p}=W_{p}^{(1-\theta) s_{0}+\theta s_{1}}(\mathbb{R}) .
$$

(v) $\left.W_{p}^{s}(\mathbb{R}) \hookrightarrow W_{q}^{t}(\mathbb{R})\right)$ if $1<p \leq q<\infty$ and $s-1 / p \geq t-1 / q$.

Besides, we need also the following properties.

(a) Given $r \in[0,1)$ and $p \in(1, \infty)$, there exists a constant $C>0$ such that

$\|g h\|_{W_{p}^{r}} \leq 2\|g\|_{\infty}\|h\|_{W_{p}^{r}}+C\|g\|_{W_{p}^{r+1}}\|h\|_{p}, \quad g \in W_{p}^{r+1}(\mathbb{R}), h \in W_{p}^{r}(\mathbb{R})$.

(b) Given $r \in(1 / p, 1)$ and $p \in(1, \infty)$, there exists a constant $C>0$ such that

$$
\|g h\|_{W_{p}^{r}} \leq 2\left(\|g\|_{\infty}\|h\|_{W_{p}^{r}}+\|h\|_{\infty}\|g\|_{W_{p}^{r}}\right), \quad g, h \in W_{p}^{r}(\mathbb{R}) .
$$


(c) Given $p \in(1,2], r \in(1 / p, 1)$, and $\rho \in(0, \min \{r-1 / p, 1-r\})$, there exists a constant $C>0$ such that

$$
\|g h\|_{W_{p^{\prime}}^{1-r}} \leq 2\|g\|_{\infty}\|h\|_{W_{p^{\prime}}^{1-r}}+C\|g\|_{W_{p}^{r}}\|h\|_{W_{p^{\prime}}^{1-r-\rho}}
$$

for all $g \in W_{p}^{r}(\mathbb{R})$ and $h \in W_{p^{\prime}}^{1-r}(\mathbb{R})$, and

$$
\|\varphi h\|_{W_{p}^{r-1}} \leq 5\|\varphi\|_{\infty}\|h\|_{W_{p}^{r-1}}+C \frac{\|\varphi\|_{W_{p}^{r}}^{1+2 r / \rho}}{\|\varphi\|_{\infty}^{2 r / \rho}}\|h\|_{W_{p}^{r-1-\rho}}
$$

for all $h \in W_{p}^{r-1}(\mathbb{R})$ and $0 \neq \varphi \in W_{p}^{r}(\mathbb{R})$.

The estimates (4) and (5) are straightforward consequences of the properties (i)-(v) listed above. The inequalities (6) and (7) are established in Appendix 2. Let us point out that (6) implies in particular that the multiplications

$$
\begin{aligned}
& {[(g, h) \mapsto g h]: W_{p}^{r}(\mathbb{R}) \times W_{p^{\prime}}^{1-r}(\mathbb{R}) \rightarrow W_{p^{\prime}}^{1-r}(\mathbb{R}),} \\
& {[(g, h) \mapsto g h]: W_{p}^{r}(\mathbb{R}) \times W_{p}^{r-1}(\mathbb{R}) \rightarrow W_{p}^{r-1}(\mathbb{R})}
\end{aligned}
$$

are both continuous if $p \in(1,2]$ and $r \in(1 / p, 1)$. The continuity of $(8)_{1}$ is a straightforward consequence of (6), while the continuity of $(8)_{2}$ follows by a standard duality argument.

\subsection{Outline}

In Sect. 2 we introduce some multilinear singular integral operators and study their properties. These operators are then used in Sect. 3 to formulate (1a) as a quasilinear evolution problem, cf. (27)-(28). Subsequently, we show in Theorem 3.5 that (27) is of parabolic type and we complete the section with the proof of Theorem 1.1. In Appendixes 1 and 2 we prove some technical results used in the analysis.

\section{Some singular integral operators}

In this section we investigate a family of multilinear singular integral operators which play a key role in the analysis of the Muskat problem (and also of the Stokes problem [27]). Given $n, m \in \mathbb{N}$ and Lipschitz continuous functions $a_{1}, \ldots, a_{m}, b_{1}, \ldots, b_{n}: \mathbb{R} \rightarrow \mathbb{R}$ we set

$$
B_{n, m}\left(a_{1}, \ldots, a_{m}\right)\left[b_{1}, \ldots, b_{n}, \bar{\omega}\right](x):=\mathrm{PV} \int_{\mathbb{R}} \frac{\bar{\omega}(x-y)}{y} \frac{\prod_{i=1}^{n}\left(\delta_{[x, y]} b_{i} / y\right)}{\prod_{i=1}^{m}\left[1+\left(\delta_{[x, y]} a_{i} / y\right)^{2}\right]} d y .
$$

For brevity we write

$$
B_{n, m}^{0}(f)[\bar{\omega}]:=B_{n, m}(f, \ldots, f)[f, \ldots, f, \bar{\omega}] .
$$


The relevance of these operators in the context of (1) is enlightened by the fact that (1a) can be recast, at least at formal level, in a compact form as

$$
\frac{d f(t)}{d t}=\frac{k}{2 \mu} \mathbb{B}(f(t))\left[\left(\sigma \kappa(f(t))-\Delta_{\rho} f(t)\right)^{\prime}\right], \quad t>0,
$$

where $\mathbb{B}(f)$ is defined by

$$
\mathbb{B}(f):=\pi^{-1}\left(B_{0,1}^{0}(f)+f^{\prime} B_{1,1}^{0}(f)\right)
$$

and $f^{\prime}:=d f / d x$. A key observation that we exploit in our analysis is the quasilinear structure of the curvature operator. Indeed, it holds that

$$
\kappa(f)=\kappa(f)[f],
$$

where

$$
\kappa(f)[h]:=\frac{h^{\prime \prime}}{\left(1+f^{\prime 2}\right)^{3 / 2}} .
$$

Given $f \in W_{p}^{s}(\mathbb{R})$ and $h \in W_{p}^{s+1}(\mathbb{R})$, with $p \in(1, \infty)$ and $s \in(1+1 / p, 2)$, Lemma 3.1 ensures that in (11) the term

$$
\left(\sigma \kappa(f)[h]-\Delta_{\rho} h\right)^{\prime}
$$

belongs to $W_{p}^{s-2}(\mathbb{R})$. Therefore, it is natural to ask weather $\mathbb{B}(f) \in \mathcal{L}\left(W_{p}^{s-2}(\mathbb{R})\right)$. The proof of this boundedness property, which enables us to view (11) as an evolution equation in $W_{p}^{s-2}(\mathbb{R})$, see Sect. 3 , is the main goal of this section. As already mentioned, some of the arguments require $p \in(1,2]$.

We first recall the following result.

Lemma 2.1 Let $p \in(1, \infty), n, m \in \mathbb{N}$, and let $a_{1}, \ldots, a_{m}, b_{1}, \ldots, b_{n}: \mathbb{R} \rightarrow \mathbb{R}$ be Lipschitz continuous. Then, there exists a constant $C=C\left(n, m, \max _{i=1, \ldots, m}\left\|a_{i}^{\prime}\right\|_{\infty}\right)$ such that

$$
\left\|B_{n, m}\left(a_{1}, \ldots, a_{m}\right)\left[b_{1}, \ldots, b_{n}, \cdot\right]\right\|_{\mathcal{L}\left(L_{p}(\mathbb{R})\right)} \leq C \prod_{i=1}^{n}\left\|b_{i}^{\prime}\right\|_{\infty} .
$$

Moreover, ${ }^{1} B_{n, m} \in \mathrm{C}^{1-}\left(\left(W_{\infty}^{1}(\mathbb{R})\right)^{m}, \mathcal{L}_{\text {sym }}^{n}\left(W_{\infty}^{1}(\mathbb{R}), \mathcal{L}\left(L_{p}(\mathbb{R})\right)\right)\right.$.

\footnotetext{
${ }^{1}$ Given $n \in \mathbb{N}$ and Banach spaces $X$ and $Y$, we let $\mathcal{L}_{\text {sym }}^{n}(X, Y)$ denote the space of $n$-linear, bounded symmetric maps $A: X^{n} \longrightarrow Y$.
} 
Proof See [2, Lemma 2].

We point out that, given Lipschitz continuous functions $a_{1}, \ldots, a_{m}, \widetilde{a}_{1}, \ldots, \widetilde{a}_{m}, b_{1}, \ldots, b_{n}$, we have

$$
\begin{aligned}
& B_{n, m}\left(\widetilde{a}_{1}, \ldots, \tilde{a}_{m}\right)\left[b_{1}, \ldots, b_{n}, \cdot\right]-B_{n, m}\left(a_{1}, \ldots, a_{m}\right)\left[b_{1}, \ldots, b_{n}, \cdot\right] \\
& =\sum_{i=1}^{m} B_{n+2, m+1}\left(\tilde{a}_{1}, \ldots, \tilde{a}_{i}, a_{i}, \ldots \ldots, a_{m}\right)\left[b_{1}, \ldots, b_{n}, a_{i}+\widetilde{a}_{i}, a_{i}-\widetilde{a}_{i}, \cdot\right] .
\end{aligned}
$$

The formula (14) was used to establish the Lipschitz continuity property (denoted by $\mathrm{C}^{1-}$ ) in Lemma 2.1 and is also of importance for our later analysis.

The strategy is as follows. In Lemma 2.4 we show that, given $f \in W_{p}^{s}(\mathbb{R})$, with $p \in(1,2]$ and $s \in(1+1 / p, 2)$, we have $B_{n, m}^{0}(f) \in \mathcal{L}\left(W_{p^{\prime}}^{r}(\mathbb{R})\right)$ for all $r \in[0,1-1 / p)$ (in particular also for $r=2-s$ ). Lemma 2.2 below provides the key argument in the proof of Lemma 2.4. The desired mapping property $B_{n, m}^{0}(f) \in \mathcal{L}\left(W_{p}^{s-2}(\mathbb{R})\right)$ stated in Lemma 2.5, follows then from Lemma 2.4 via a duality argument. Lemma 2.5 and the fact that $f^{\prime} \in W_{p}^{s-1}(\mathbb{R})$ is a pointwise multiplier for $W_{p}^{s-2}(\mathbb{R})$, see (8), provide the desired property $\mathbb{B}(f) \in \mathcal{L}\left(W_{p}^{s-2}(\mathbb{R})\right)$.

Lemma 2.2 Given $p \in(1,2], s \in(1+1 / p, 2), r \in(0,1-1 / p), n, m \in \mathbb{N}, n \geq 1$, and $a_{1}, \ldots, a_{m} \in W_{p}^{s}(\mathbb{R})$, there exists a positive constant $C$, which depends only on $n, m, s, r, p, \max _{1 \leq i \leq m}\left\|a_{i}\right\|_{W_{p}^{s}}$, such that

$$
\left\|B_{n, m}\left(a_{1}, \ldots, a_{m}\right)\left[b_{1}, \ldots, b_{n}, \bar{\omega}\right]\right\|_{p^{\prime}} \leq C\left\|b_{1}\right\|_{W_{p^{\prime}}^{s+1-r-2 / p}}\|\bar{\omega}\|_{W_{p^{\prime}}^{r}} \prod_{i=2}^{n}\left\|b_{i}\right\|_{W_{p}^{s}}
$$

for all $b_{1}, \ldots, b_{n} \in W_{p}^{s}(\mathbb{R})$ and $\bar{\omega} \in W_{p^{\prime}}^{r}(\mathbb{R})$.

Proof Without loss of generality we may assume that $\bar{\omega} \in \mathrm{C}_{0}^{\infty}(\mathbb{R})$. Using the relation

$$
\frac{\partial}{\partial y}\left(\frac{\delta_{[x, y]} b_{1}}{y}\right)=\frac{b_{1}^{\prime}(x-y)}{y}-\frac{\delta_{[x, y]} b_{1}}{y^{2}}
$$

algebraic manipulations lead us to

$$
\begin{aligned}
& B_{n, m}\left(a_{1}, \ldots, a_{m}\right)\left[b_{1}, \ldots, b_{n}, \bar{\omega}\right](x) \\
& =\left(\bar{\omega} B_{n-1, m}\left(a_{1}, \ldots, a_{m}\right)\left[b_{2}, \ldots, b_{n}, b_{1}^{\prime}\right]\right)(x)+\int_{\mathbb{R}} K(x, y) d y \\
& -\bar{\omega}(x) \int_{\mathbb{R}} \frac{\prod_{i=2}^{n}\left(\delta_{[x, y]} b_{i} / y\right)}{\prod_{i=1}^{m}\left[1+\left(\delta_{[x, y]} a_{i} / y\right)^{2}\right]} \frac{\partial}{\partial y}\left(\frac{\delta_{[x, y]} b_{1}}{y}\right) d y
\end{aligned}
$$

for $x \in \mathbb{R}$, where 


$$
K(x, y):=-\frac{\prod_{i=1}^{n}\left(\delta_{[x, y]} b_{i} / y\right)}{\prod_{i=1}^{m}\left[1+\left(\delta_{[x, y]} a_{i} / y\right)^{2}\right]} \frac{\delta_{[x, y]} \bar{\omega}}{y}, \quad x \in \mathbb{R}, y \neq 0 .
$$

The last term on the right side of the previous identity vanishes if $(n-1)^{2}+m^{2}=0$. Otherwise, we use integration by parts and arrive at

$$
\begin{aligned}
& B_{n, m}\left(a_{1}, \ldots, a_{m}\right)\left[b_{1}, \ldots, b_{n}, \bar{\omega}\right](x) \\
& =\left(\bar{\omega} B_{n-1, m}\left(a_{1}, \ldots, a_{m}\right)\left[b_{2}, \ldots, b_{n}, b_{1}^{\prime}\right]\right)(x)+\int_{\mathbb{R}} K(x, y) d y \\
& -\bar{\omega}(x) \sum_{j=2}^{n} \int_{\mathbb{R}} K_{1, j}(x, y) d y+\bar{\omega}(x) \sum_{j=1}^{m} \int_{\mathbb{R}} K_{2, j}(x, y) d y,
\end{aligned}
$$

where, given $x \in \mathbb{R}$ and $y \neq 0$, we set

$$
\begin{aligned}
K_{1, j}(x, y) & :=\frac{\prod_{i=1, i \neq j}^{n}\left(\delta_{[x, y]} b_{i} / y\right)}{\prod_{i=1}^{m}\left[1+\left(\delta_{[x, y]} a_{i} / y\right)^{2}\right]} \frac{\delta_{[x, y]} b_{j}-y b_{j}^{\prime}(x-y)}{y^{2}}, \\
K_{2, j}(x, y) & :=2 \frac{\delta_{[x, y]} a_{j} / y}{1+\left(\delta_{[x, y]} a_{j} / y\right)^{2}} \frac{\prod_{i=1}^{n}\left(\delta_{[x, y]} b_{i} / y\right)}{\prod_{i=1}^{m}\left[1+\left(\delta_{[x, y]} a_{i} / y\right)^{2}\right]} \frac{\delta_{[x, y]} a_{j}-y a_{j}^{\prime}(x-y)}{y^{2}} .
\end{aligned}
$$

We estimate the $L_{p^{\prime}}$-norm of the four terms on the right of (16) separately. Let $q \in\left(p^{\prime}, \infty\right)$ be defined as the solution to

$$
\frac{1}{q}+\frac{1}{1 / r}=\frac{1}{p^{\prime}}
$$

Term 1. We note that $\bar{\omega} \in W_{p^{\prime}}^{r}(\mathbb{R}) \hookrightarrow L_{q}(\mathbb{R}), b_{1}^{\prime} \in W_{p^{\prime}}^{s-2 / p}(\mathbb{R}) \hookrightarrow W_{p^{\prime}}^{s-r-2 / p}(\mathbb{R})$, and additionally we have $W_{p^{\prime}}^{s-r-2 / p}(\mathbb{R}) \hookrightarrow L_{1 / r}(\mathbb{R})$. Hölder's inequality together with Lemma 2.1 (with $p=1 / r$ ) then yields

$$
\begin{aligned}
\left\|\bar{\omega} B_{n-1, m}\left(a_{1}, \ldots, a_{m}\right)\left[b_{2}, \ldots, b_{n}, b_{1}^{\prime}\right]\right\|_{p^{\prime}} & \leq\|\bar{\omega}\|_{q}\left\|B_{n-1, m}\left(a_{1}, \ldots, a_{m}\right)\left[b_{2}, \ldots, b_{n}, b_{1}^{\prime}\right]\right\|_{1 / r} \\
& \leq C\|\bar{\omega}\|_{q}\left\|b_{1}^{\prime}\right\|_{1 / r}\left(\prod_{i=2}^{n}\left\|b_{i}^{\prime}\right\|_{\infty}\right) \\
& \leq C\|\bar{\omega}\|_{W_{p^{\prime}}^{r}}\left\|b_{1}\right\|_{W_{p^{\prime}}^{s+1-r-2 / p}}\left(\prod_{i=2}^{n}\left\|b_{i}\right\|_{W_{p}^{s}}\right) .
\end{aligned}
$$

Term 2. Let $s_{0} \in(1+1 / p, s]$ be chosen such that $s_{0}-r-1 / p<1$. Taking advantage of Minkowski's integral inequality, of Hölder's inequality, and of the embedding property $b_{1} \in W_{p^{\prime}}^{s+1-r-2 / p}(\mathbb{R}) \hookrightarrow C^{s_{0}-r-1 / p}(\mathbb{R})$ we get 


$$
\begin{aligned}
\left(\int_{\mathbb{R}}\left|\int_{\mathbb{R}} K(x, y) d y\right|^{p^{\prime}} d x\right)^{1 / p^{\prime}} & \leq\left(\int_{\{|y|<1\}}+\int_{\{|y|>1\}}\right)\left(\int_{\mathbb{R}}|K(x, y)|^{p^{\prime}} d x\right)^{1 / p^{\prime}} d y \\
& \leq 4\left\|b_{1}\right\|_{\infty}\left(\prod_{i=2}^{n}\left\|b_{i}^{\prime}\right\|_{\infty}\right)\|\bar{\omega}\|_{p^{\prime}} \\
& +\left[b_{1}\right]_{s_{0}-r-1 / p}\left(\prod_{i=2}^{n}\left\|b_{i}^{\prime}\right\|_{\infty}\right) \int_{\{|y|<1\}} \frac{\left\|\bar{\omega}-\tau_{y} \bar{\omega}\right\|_{p^{\prime}}}{|y|^{2-s_{0}+r+1 / p}} d y,
\end{aligned}
$$

where

$$
\int_{\{|y|<1\}} \frac{\left\|\bar{\omega}-\tau_{y} \bar{\omega}\right\|_{p^{\prime}}}{|y|^{2-s_{0}+r+1 / p}} d y \leq\|\bar{\omega}\|_{W_{p^{\prime}}}\left(\int_{\{|y|<1\}} \frac{1}{|y|^{\left(1-s_{0}+2 / p\right) p}} d y\right)^{1 / p} \leq C\|\bar{\omega}\|_{W_{p^{\prime}}^{r}} .
$$

We arrive at

$$
\left(\int_{\mathbb{R}}\left|\int_{\mathbb{R}} K(x, y) d y\right|^{p^{\prime}} d x\right)^{1 / p^{\prime}} \leq C\|\bar{\omega}\|_{W_{p^{\prime}}^{r}}\left\|b_{1}\right\|_{W_{p^{\prime}}^{s+1-r-2 / p}}\left(\prod_{i=2}^{n}\left\|b_{i}\right\|_{W_{p}^{s}}\right) .
$$

Terms $3 \& 4$. Given $2 \leq j \leq n$, Hölder inequality and Minkowski's integral inequality yield

$$
\begin{aligned}
\left(\int_{\mathbb{R}}\left|\bar{\omega}(x) \int_{\mathbb{R}} K_{1, j}(x, y) d y\right|^{p^{\prime}} d x\right)^{1 / p^{\prime}} & \leq\|\bar{\omega}\|_{q}\left(\int_{\mathbb{R}}\left|\int_{\mathbb{R}} K_{1, j}(x, y) d y\right|^{\frac{1}{r}} d x\right)^{r} \\
& \leq\|\bar{\omega}\|_{q} \int_{\mathbb{R}}\left(\int_{\mathbb{R}}\left|K_{1, j}(x, y)\right|^{\frac{1}{r}} d x\right)^{r} d y .
\end{aligned}
$$

To estimate the integral term we choose $s_{0} \in(1+1 / p, s]$ with $s_{0}-r-1 / p<1$. Since $b_{1} \in W_{p^{\prime}}^{s+1-r-2 / p}(\mathbb{R}) \hookrightarrow W_{1 / r}^{1}(\mathbb{R}) \cap C^{s_{0}-r-1 / p}(\mathbb{R})$, we get

$$
\begin{aligned}
& \int_{\mathbb{R}}\left(\int_{\mathbb{R}}\left|K_{1, j}(x, y)\right|^{1 / r} d x\right)^{r} d y \\
& \leq 4\left\|b_{1}\right\|_{1 / r}\left(\prod_{i=2}^{n}\left\|b_{i}\right\|_{W_{p}^{s}}\right) \\
& +\left[b_{1}\right]_{s_{0}-r-1 / p}\left(\prod_{i=2, i \neq j}^{n}\left\|b_{i}^{\prime}\right\|_{\infty}\right) \int_{\{|y|<1\}} \frac{\left\|b_{j}-\tau_{y} b_{j}-y \tau_{y} b_{j}^{\prime}\right\|_{1 / r}}{|y|^{3-s_{0}+r+1 / p}} d y
\end{aligned}
$$

for $2 \leq j \leq n$. Since $b_{j} \in W_{p}^{s_{0}}(\mathbb{R}) \hookrightarrow W_{1 / r}^{s_{0}+r-\frac{1}{p}}(\mathbb{R}), 2 \leq j \leq n$, Minkowski's integral inequality, Hölder's inequality, and a change of variables lead to 
644

A.-V. Matioc, B.-V. Matioc

$$
\begin{aligned}
\int_{\{|y|<1\}} & \frac{\left\|b_{j}-\tau_{y} b_{j}-y \tau_{y} b_{j}^{\prime}\right\|_{1 / r}}{|y|^{3-s_{0}+r+1 / p}} d y \\
& =\int_{\{|y| 1\}} \frac{1}{|y|^{2-s_{0}+r+1 / p}}\left(\int_{\mathbb{R}}\left|\int_{0}^{1}\left[b_{j}^{\prime}(x-(1-t) y)-b_{j}^{\prime}(x-y)\right] d t\right|^{1 / r} d x\right)^{r} d y \\
& \leq \int_{0}^{1} \int_{\{|y|<1\}} \frac{\left\|b_{j}^{\prime}-\tau_{-t y} b_{j}^{\prime}\right\|_{1 / r}}{|y|^{2-s_{0}+r+1 / p}} d y d t \\
& \leq\left(\int_{\{|y|<1\}} \frac{1}{|y|^{\left(3-2 s_{0}-r+2 / p\right) /(1-r)}} d y\right)^{1-r}\left(\int_{0}^{1} t^{1-s_{0}+r+1 / p} d t\right)\left\|b_{j}^{\prime}\right\|_{W_{1 / r}^{s_{0}+r-1-\frac{1}{p}}} \\
& \leq C\left\|b_{j}\right\|_{W_{p}^{s} .}
\end{aligned}
$$

Consequently, given $2 \leq j \leq n$, we have

$$
\left(\int_{\mathbb{R}}\left|\bar{\omega}(x) \int_{\mathbb{R}} K_{1, j}(x, y) d y\right|^{p^{\prime}} d x\right)^{1 / p^{\prime}} \leq C\|\bar{\omega}\|_{W_{p^{\prime}}^{r}}\left\|b_{1}\right\|_{W_{p^{\prime}}^{s+1-r-2 / p}}\left(\prod_{i=2}^{n}\left\|b_{i}\right\|_{W_{p}^{s}}\right)
$$

and, similarly,

$$
\left(\int_{\mathbb{R}}\left|\bar{\omega}(x) \int_{\mathbb{R}} K_{2, j}(x, y) d y\right|^{p^{\prime}} d x\right)^{1 / p^{\prime}} \leq C\|\bar{\omega}\|_{W_{p^{\prime}}^{r}}\left\|b_{1}\right\|_{W_{p^{\prime}}^{s+1-r-2 / p}}\left(\prod_{i=2}^{n}\left\|b_{i}\right\|_{W_{p}^{s}}\right) .
$$

The desired claim follows now from (16) to (20).

Lemma 2.3 below is used to prove Lemma 2.4 (see also [2, Lemma 7] for a related result).

Lemma 2.3 Let $p \in(1, \infty), n \in \mathbb{N}$, and $n<t^{\prime}<t<n+1$. Then, there exists $C>0$ such that

$$
\int_{\mathbb{R}} \frac{\left\|b-\tau_{\xi} b\right\|_{W_{p}^{t^{\prime}}}^{p}}{|\xi|^{1+\left(t-t^{\prime}\right) p}} d \xi \leq C\|b\|_{W_{p}^{t}}^{p} \quad \text { for all } \quad b \in W_{p}^{t}(\mathbb{R}) .
$$

Proof We have

$$
\left\|b-\tau_{\xi} b\right\|_{W_{p}^{\prime^{\prime}}}^{p}=\sum_{k=0}^{n}\left\|b^{(k)}-\tau_{\xi} b^{(k)}\right\|_{p}^{p}+\left[b-\tau_{\xi} b\right]_{W_{p}^{\prime^{\prime}}}^{p}
$$

and, given $k \in\{0, \ldots, n\}$,

Springer 


$$
\int_{\mathbb{R}} \frac{\left\|b^{(k)}-\tau_{\xi} b^{(k)}\right\|_{p}^{p}}{|\xi|^{1+\left(t-t^{\prime}\right) p}} d \xi=\left[b^{(k)}\right]_{W_{p}^{t-t^{\prime}}}^{p} \leq C\|b\|_{W_{p}^{t}}^{p} .
$$

Moreover, since $\mathbb{R}^{2}=\overline{\{|\xi|<|h|\}} \cup\{|\xi|>|h|\}$ and

$$
\int_{\mathbb{R}} \frac{\left[b-\tau_{\xi} b\right]_{W_{p}^{t^{\prime}}}^{p}}{|\xi|^{1+\left(t-t^{\prime}\right) p}} d \xi=\int_{\mathbb{R}^{2}} \frac{\left\|b^{(n)}-\tau_{\xi} b^{(n)}-\tau_{h}\left(b^{(n)}-\tau_{\xi} b^{(n)}\right)\right\|_{p}^{p}}{|\xi|^{1+\left(t-t^{\prime}\right) p}|h|^{1+\left(t^{\prime}-n\right) p}} d h d \xi,
$$

with

$$
\begin{aligned}
& \int_{\{|\xi|<|h|\}} \frac{\left\|b^{(n)}-\tau_{\xi} b^{(n)}-\tau_{h}\left(b^{(n)}-\tau_{\xi} b^{(n)}\right)\right\|_{p}^{p}}{|\xi|^{1+\left(t-t^{\prime}\right) p}|h|^{1+\left(t^{\prime}-n\right) p}} d h d \xi \\
& \leq 2^{p} \int_{\mathbb{R}} \frac{\left\|b^{(n)}-\tau_{\xi} b^{(n)}\right\|_{p}^{p}}{|\xi|^{1+\left(t-t^{\prime}\right) p}}\left(\int_{\{|\xi|<|h|\}} \frac{1}{|h|^{1+\left(t^{\prime}-n\right) p}} d h\right) d \xi \leq C[b]_{W_{p}^{t}}^{p} \\
& \int_{\{|h|<|\xi|\}} \frac{\left\|b^{(n)}-\tau_{\xi} b^{(n)}-\tau_{h}\left(b^{(n)}-\tau_{\xi} b^{(n)}\right)\right\|_{p}^{p}}{|\xi|^{1+\left(t-t^{\prime}\right) p}|h|^{1+\left(t^{\prime}-n\right) p}} d h d \xi \\
& \leq 2^{p} \int_{\mathbb{R}} \frac{\left\|b^{(n)}-\tau_{h} b^{(n)}\right\|_{p}^{p}}{|h|^{1+\left(t^{\prime}-n\right) p}}\left(\int_{\{|h|<|\xi|\}} \frac{1}{|\xi|^{1+\left(t-t^{\prime}\right) p}} d \xi\right) d h \leq C[b]_{W_{p}^{t}}^{p},
\end{aligned}
$$

the desired estimate is immediate.

We are now in a position to prove that $B_{n, m}^{0}(f) \in \mathcal{L}\left(W_{p^{\prime}}^{r}(\mathbb{R})\right)$ for all $r \in[0,1-1 / p)$.

Lemma 2.4 Given $p \in(1,2], s \in(1+1 / p, 2), n, m \in \mathbb{N}, a_{1}, \ldots, a_{m} \in W_{p}^{s}(\mathbb{R})$, and $r \in[0,1-1 / p)$, there exists a constant $C=C\left(n, m, s, r, p, \max _{1 \leq i \leq m}\left\|a_{i}\right\|_{W_{p}^{s}}\right)$ such that

$$
\left\|B_{n, m}\left(a_{1}, \ldots, a_{m}\right)\left[b_{1}, \ldots, b_{n}, \bar{\omega}\right]\right\|_{W_{p^{\prime}}^{r}} \leq C\|\bar{\omega}\|_{W_{p^{\prime}}^{r}} \prod_{i=1}^{n}\left\|b_{i}\right\|_{W_{p}^{s}}
$$

for all $b_{1}, \ldots, b_{n} \in W_{p}^{s}(\mathbb{R})$ and $\bar{\omega} \in W_{p^{\prime}}^{r}(\mathbb{R})$.

Moreover, $B_{n, m} \in \mathrm{C}^{1-}\left(\left(W_{p}^{s}(\mathbb{R})\right)^{m}, \mathcal{L}_{\text {sym }}^{n}\left(W_{p}^{s}(\mathbb{R}), \mathcal{L}\left(W_{p^{\prime}}^{r}(\mathbb{R})\right)\right)\right)$.

Proof Let $B_{n, m}:=B_{n, m}\left(a_{1}, \ldots, a_{m}\right)\left[b_{1}, \ldots, b_{n}, \cdot\right]$. Recalling Lemma 2.1 (with $p=p^{\prime}$ ), we get

$$
\left\|B_{n, m}[\bar{\omega}]\right\|_{p^{\prime}} \leq C\|\bar{\omega}\|_{p^{\prime}} \prod_{i=1}^{n}\left\|b_{i}\right\|_{W_{p}^{s}}
$$


which proves (21) for $r=0$. Let now $r \in(0,1-1 / p)$. It remains to estimate the quantity

$$
\left[B_{n, m}[\bar{\omega}]\right]_{W_{p^{\prime}}^{p^{\prime}}}^{p^{\prime}}=\int_{\mathbb{R}} \frac{\left\|B_{n, m}[\bar{\omega}]-\tau_{\xi} B_{n, m}[\bar{\omega}]\right\|_{p^{\prime}}^{p^{\prime}}}{|\xi|^{1+r p^{\prime}}} d \xi
$$

Taking advantage of (14), we write

$$
B_{n, m}[\bar{\omega}]-\tau_{\xi} B_{n, m}[\bar{\omega}]=T_{1}(\cdot, \xi)+T_{2}(\cdot, \xi)-T_{3}(\cdot, \xi), \quad \xi \in \mathbb{R}
$$

with

$$
\begin{aligned}
T_{1}(\cdot, \xi) & :=B_{n, m}\left(a_{1}, \ldots, a_{m}\right)\left[b_{1}, \ldots, b_{n}, \bar{\omega}-\tau_{\xi} \bar{\omega}\right], \\
T_{2}(\cdot, \xi) & :=\sum_{i=1}^{n} B_{n, m}\left(a_{1}, \ldots, a_{m}\right)\left[\tau_{\xi} b_{1}, \ldots, \tau_{\xi} b_{i-1}, b_{i}-\tau_{\xi} b_{i}, b_{i+1}, \ldots b_{n}, \tau_{\xi} \bar{\omega}\right], \\
T_{3}(\cdot, \xi) & :=\sum_{i=1}^{m} B_{n+2, m+1}\left(a_{1}, \ldots, a_{i}, \tau_{\xi} a_{i}, \ldots, \tau_{\xi} a_{m}\right)\left[\tau_{\xi} b_{1}, \ldots \tau_{\xi} b_{n}, a_{i}+\tau_{\xi} a_{i}, a_{i}-\tau_{\xi} a_{i}, \tau_{\xi} \bar{\omega}\right] .
\end{aligned}
$$

Hence,

$$
\left[B_{n, m}[\bar{\omega}]\right]_{W_{p^{\prime}}^{p^{\prime}}}^{p^{\prime}} \leq 3^{p^{\prime}} \sum_{\ell=1}^{3} \int_{\mathbb{R}} \frac{\left\|T_{\ell}(\cdot, \xi)\right\|_{p^{\prime}}^{p^{\prime}}}{|\xi|^{1+r p^{\prime}}} d \xi
$$

and Lemma 2.1 (with $p=p^{\prime}$ ) yields

$$
\int_{\mathbb{R}} \frac{\left\|T_{1}(\cdot, \xi)\right\|_{p^{\prime}}^{p^{\prime}}}{|\xi|^{1+r p^{\prime}}} d \xi \leq C^{p}\left(\prod_{i=1}^{n}\left\|b_{i}^{\prime}\right\|_{\infty}^{p^{\prime}}\right) \int_{\mathbb{R}} \frac{\left\|\bar{\omega}-\tau_{\xi} \bar{\omega}\right\|_{p^{\prime}}^{p^{\prime}}}{|\xi|^{1+r p^{\prime}}} d \xi \leq\left(C[\bar{\omega}]_{W_{p^{\prime}}} \prod_{i=1}^{n}\left\|b_{i}^{\prime}\right\|_{\infty}\right)^{p^{\prime}} .
$$

Furthermore, using (15), Lemma 2.3 (with $p=p^{\prime}, \quad t=s+1-2 / p$, and $\left.t^{\prime}=s+1-r-2 / p\right)$, and the embedding $W_{p}^{s}(\mathbb{R}) \hookrightarrow W_{p^{\prime}}^{s+1-2 / p}(\mathbb{R})$, we deduce that

$$
\begin{aligned}
\int_{\mathbb{R}} \frac{\left\|T_{2}(\cdot, \xi)\right\|_{p^{\prime}}^{p^{\prime}}}{|\xi|^{1+r p^{\prime}}} d \xi & \leq C^{p}\|\bar{\omega}\|_{W_{p^{\prime}}^{p^{\prime}}}^{p^{\prime}} \sum_{i=1}^{n}\left(\int_{\mathbb{R}} \frac{\left\|b_{i}-\tau_{\xi} b_{i}\right\|_{W_{p^{\prime}}^{t^{\prime}}}^{p^{\prime}}}{|\xi|^{1+\left(t-t^{\prime}\right) p^{\prime}}} d \xi\right) \prod_{j=1, j \neq i}^{n}\left\|b_{j}\right\|_{W_{p}^{s}}^{p^{\prime}} \\
& \leq\left(C\|\bar{\omega}\|_{W_{p^{\prime}}^{r}} \prod_{i=1}^{n}\left\|b_{i}\right\|_{W_{p}^{s}}\right)^{p^{\prime}}
\end{aligned}
$$

and, by similar arguments,

$$
\int_{\mathbb{R}} \frac{\left\|T_{3}(\cdot, \xi)\right\|_{p^{\prime}}^{p^{\prime}}}{|\xi|^{1+r p^{\prime}}} d \xi \leq\left(C\|\bar{\omega}\|_{W_{p^{\prime}}^{r}} \prod_{i=1}^{n}\left\|b_{i}\right\|_{W_{p}^{s}}\right)^{p^{\prime}}
$$


The relations (22)-(25) lead to the desired estimate. The local Lipschitz continuity property follows from (14) and (21).

Together with Lemma 2.4 (with $r=2-s \in(0,1-1 / p)$ ) we obtain the following result.

Lemma 2.5 Given $p \in(1,2], s \in(1+1 / p, 2), n, m \in \mathbb{N}$, and $a_{1}, \ldots, a_{m} \in W_{p}^{s}(\mathbb{R})$, there exists a constant $C=C\left(n, m, s, p, \max _{1 \leq i \leq m}\left\|a_{i}\right\|_{W_{p}^{s}}\right)$ such that

$$
\left\|B_{n, m}\left(a_{1}, \ldots, a_{m}\right)\left[b_{1}, \ldots, b_{n}, \bar{\omega}\right]\right\|_{W_{p}^{s-2}} \leq C\|\bar{\omega}\|_{W_{p}^{s-2}} \prod_{i=1}^{n}\left\|b_{i}\right\|_{W_{p}^{s}}
$$

for all $b_{1}, \ldots, b_{n} \in W_{p}^{s}(\mathbb{R})$ and $\bar{\omega} \in L_{p}(\mathbb{R})$.

Moreover, $B_{n, m} \in \mathrm{C}^{1-}\left(\left(W_{p}^{s}(\mathbb{R})\right)^{m}, \mathcal{L}_{\mathrm{sym}}^{n}\left(W_{p}^{s}(\mathbb{R}), \mathcal{L}\left(W_{p}^{s-2}(\mathbb{R})\right)\right)\right)$.

Proof We recall that $W_{p}^{s-2}(\mathbb{R})=\left(W_{p^{\prime}}^{2-s}(\mathbb{R})\right)^{\prime}$. Let $B_{n, m}:=B_{n, m}\left(a_{1}, \ldots, a_{m}\right)\left[b_{1}, \ldots, b_{n}, \cdot\right]$. It is not difficult to prove that the $L_{2}$-adjoint of $B_{n, m}$ is the operator $-B_{n, m}$. Therefore, given $\bar{\omega}, \varphi \in \mathrm{C}_{0}^{\infty}(\mathbb{R})$, we obtain, in view of Lemma 2.4 ,

$$
\begin{aligned}
\left|\left\langle B_{n, m}[\bar{\omega}] \mid \varphi\right\rangle_{W_{p}^{s-2}(\mathbb{R}) \times W_{p^{\prime}}^{2-s}(\mathbb{R})}\right| & =\left|\left\langle\bar{\omega} \mid B_{n, m}[\varphi]\right\rangle_{W_{p}^{s-2}(\mathbb{R}) \times W_{p^{\prime}}^{2-s}(\mathbb{R})}\right| \\
& \leq C\|\bar{\omega}\|_{W_{p}^{s-2}}\|\varphi\|_{W_{p^{\prime}}^{2-s}} \prod_{i=1}^{n}\left\|b_{i}\right\|_{W_{p}^{s}} .
\end{aligned}
$$

The estimate (26) follows via a standard density argument.The Lipschitz continuity property is a consequence of (26) and of (14).

\section{A functional analytic framework for the Muskat problem}

In this section we take advantage of the mapping properties established in Sect. 2 and formulate the Muskat problem (1a) as a quasilinear evolution problem in a suitable functional analytic setting, see (27)-(29). Afterwards, we show that the problem is of parabolic type. This enables us to employ theory for such evolution equations as presented in $[6,28]$ to establish our main result in Theorem 1.1. The quasilinear structure of (1a) is due to the quasilinearity of the curvature operator, the latter being established in Lemma 3.1.

Lemma 3.1 Given $p \in(1, \infty)$ and $s \in(1+1 / p, 2)$, the operator $\kappa(\cdot)[\cdot]$ defined in (13) satisfies $\kappa \in \mathrm{C}^{\infty}\left(W_{p}^{s}(\mathbb{R}), \mathcal{L}\left(W_{p}^{s+1}(\mathbb{R}), W_{p}^{s-1}(\mathbb{R})\right)\right)$.

Proof The arguments are similar to those presented in [27, Appendix C] and are therefore omitted. 
The Muskat problem (1a) can thus be formulated as the evolution problem

$$
\frac{d f}{d t}(t)=\Phi(f(t))[f(t)], \quad t>0, \quad f(0)=f_{0},
$$

where

$$
\Phi(f)[h]:=\frac{k}{2 \mu} \mathbb{B}(f)\left[\sigma(\kappa(f)[h])^{\prime}-\Delta_{\rho} h^{\prime}\right]
$$

with $\mathbb{B}$ introduced in (12). Arguing as in [27, Appendix $C]$, we may infer from Lemma 2.5 that, given $n, m \in \mathbb{N}, p \in(1,2]$, and $s \in(1+1 / p, 2)$, we have

$$
\left[f \mapsto B_{n, m}^{0}(f)\right] \in \mathrm{C}^{\infty}\left(W_{p}^{s}(\mathbb{R}), \mathcal{L}\left(W_{p}^{s-2}(\mathbb{R})\right)\right)
$$

This property and Lemma 3.1 combined yield

$$
\Phi \in \mathrm{C}^{\infty}\left(W_{p}^{s}(\mathbb{R}), \mathcal{L}\left(W_{p}^{s+1}(\mathbb{R}), W_{p}^{s-2}(\mathbb{R})\right)\right)
$$

for all $p \in(1,2]$ and $s \in(1+1 / p, 2)$.

Let $p \in(1,2], s \in(1+1 / p, 2)$, and $f \in W_{p}^{s}(\mathbb{R})$ be fixed in the remaining of this section. The analysis below is devoted to showing that the linear operator $\Phi(f)$, viewed as an unbounded operator in $W_{p}^{s-2}(\mathbb{R})$ and with definition domain $W_{p}^{s+1}(\mathbb{R})$, is the generator of an analytic semigroup in $\mathcal{L}\left(W_{p}^{2-s}(\mathbb{R})\right)$, which reads in the notation used in [7] as

$$
-\Phi(f) \in \mathcal{H}\left(W_{p}^{s+1}(\mathbb{R}), W_{p}^{s-2}(\mathbb{R})\right) .
$$

This property is established in Theorem 3.5 below and it identifies the quasilinear evolution problem (27) as being of parabolic type. To start, we note that $\pi^{-1} B_{0,0}=H$, where $H$ is the Hilbert transform, and therefore

$$
\Phi(0)=\frac{k \sigma}{2 \mu} H \circ \frac{d^{3}}{d x^{3}}-\frac{k \Delta \rho}{2 \mu} H \circ \frac{d}{d x}=-\frac{k \sigma}{2 \mu}\left(\frac{d^{4}}{d x^{4}}\right)^{3 / 4}-\frac{k \Delta \rho}{2 \mu}\left(-\frac{d^{2}}{d x^{2}}\right)^{1 / 2},
$$

where $\left(d^{4} / d x^{4}\right)^{3 / 4}$ denotes the Fourier multiplier with symbol $m(\xi):=|\xi|^{3}$ and $\left(-d^{2} / d x^{2}\right)^{1 / 2}$ is the Fourier multiplier with symbol $m(\xi):=|\xi|$. We shall locally approximate the operator $\Phi(\tau f)$, with $\tau \in[0,1]$, by certain Fourier multipliers $\mathbb{A}_{j, \tau}$. Therefore we choose for each $\varepsilon \in(0,1)$ a so-called finite $\varepsilon$-localization family, that is a set

$$
\left\{\left(\pi_{j}^{\varepsilon}, x_{j}^{\varepsilon}\right):-N+1 \leq j \leq N\right\} \subset \mathrm{C}^{\infty}(\mathbb{R},[0,1]) \times \mathbb{R}
$$

such that 
- $\sum_{j=-N+1}^{N}\left(\pi_{j}^{\varepsilon}\right)^{2}=1$ and $\left.\| \pi_{j}^{\varepsilon}\right)^{(k)} \|_{\infty} \leq C \varepsilon^{-k}$ for all $k \in \mathbb{N},-N+1 \leq j \leq N$;

- $\operatorname{supp} \pi_{j}^{\varepsilon}$ is an interval of length $\varepsilon$ for $|j| \leq N-1$, supp $\pi_{N}^{\varepsilon} \subset\{|x|>1 / \varepsilon\}$;

- $\pi_{j}^{\varepsilon} \cdot \pi_{l}^{\varepsilon}=0$ if $[|j-l| \geq 2, \max \{|j|,|l|\} \leq N-1]$ or $[|l| \leq N-2, j=N]$;

- $x_{j}^{\varepsilon} \in \operatorname{supp} \pi_{j}^{\varepsilon},|j| \leq N-1$.

The real number $x_{N}^{\varepsilon}$ plays no role in the analysis below. To each finite $\varepsilon$-localization family we associate a second family $\left\{\chi_{j}^{\varepsilon}:-N+1 \leq j \leq N\right\} \subset \mathrm{C}^{\infty}(\mathbb{R},[0,1])$ such that

- $\chi_{j}^{\varepsilon}=1$ on $\operatorname{supp} \pi_{j}^{\varepsilon},-N+1 \leq j \leq N$, and $\operatorname{supp} \chi_{N}^{\varepsilon} \subset\{|x|>1 / \varepsilon-\varepsilon\}$;

- $\operatorname{supp} \chi_{j}^{\varepsilon}$ is an interval of length $3 \varepsilon$ with the same midpoint as $\operatorname{supp} \pi_{j}^{\varepsilon},|j| \leq N-1$.

To each finite $\varepsilon$-localization family we associate a norm on $W_{p}^{r}(\mathbb{R}), r \in \mathbb{R}$, which is equivalent to the standard norm.

Lemma 3.2 Let $\varepsilon \in(0,1)$ and let $\left\{\left(\pi_{j}^{\varepsilon}, x_{j}^{\varepsilon}\right):-N+1 \leq j \leq N\right\}$ be a finite $\varepsilon$-localization family. Given $p \in(1, \infty)$ and $r \in \mathbb{R}$, there exists $c=c(\varepsilon, r, p) \in(0,1)$ such that

$$
c\|f\|_{W_{p}^{r}} \leq \sum_{j=-N+1}^{N}\left\|\pi_{j}^{\varepsilon} f\right\|_{W_{p}^{r}} \leq c^{-1}\|f\|_{W_{p}^{r}}, \quad f \in W_{p}^{r}(\mathbb{R}) .
$$

Proof The claim follows from the fact that $\pi_{j}^{\varepsilon} \in \mathrm{C}^{\infty}(\mathbb{R})$ is a pointwise multiplier for $W_{p}^{r}(\mathbb{R})$.

The next result is the main step in the proof of (30).

Theorem 3.3 Let $p \in(1,2], s \in(1+1 / p, 2), \rho \in(0, \min \{(s-1-1 / p) / 2), 2-s\})$, and $v>0$ be given. Then, there exist $\varepsilon \in(0,1), a$-localization family $\left\{\left(\pi_{j}^{\varepsilon}, x_{j}^{\varepsilon}\right):-N+1 \leq j \leq N\right\}$, a constant $K=K(\varepsilon)$, and bounded operators

$$
\mathbb{A}_{j, \tau} \in \mathcal{L}\left(W_{p}^{s+1}(\mathbb{R}), W_{p}^{s-2}(\mathbb{R})\right), \quad j \in\{-N+1, \ldots, N\} \text { and } \tau \in[0,1],
$$

such that

$$
\left\|\pi_{j}^{\varepsilon} \Phi(\tau f)[h]-\mathbb{A}_{j, \tau}\left[\pi_{j}^{\varepsilon} h\right]\right\|_{W_{p}^{s-2}} \leq v\left\|\pi_{j}^{\varepsilon} h\right\|_{W_{p}^{s+1}}+K\|h\|_{W_{p}^{s+1-\rho}}
$$

for all $-N+1 \leq j \leq N, \tau \in[0,1]$, and $h \in W_{p}^{s+1}(\mathbb{R})$. The operators $\mathbb{A}_{j, \tau}$ are defined by

$$
\mathbb{A}_{j, \tau}:=-\alpha_{\tau}\left(x_{j}^{\varepsilon}\right)\left(\frac{d^{4}}{d x^{4}}\right)^{3 / 4}, \quad|j| \leq N-1, \quad \mathbb{A}_{N, \tau}:=-\frac{k \sigma}{2 \mu}\left(\frac{d^{4}}{d x^{4}}\right)^{3 / 4},
$$


and $\alpha_{\tau}:=(k \sigma /(2 \mu))\left(1+\tau^{2} f^{\prime 2}\right)^{-3 / 2}$.

Proof In this proof we denote by $C$ constants that do not depend on $\varepsilon$ and we write $K$ for constants that depend on $\varepsilon$.

Given $-N+1 \leq j \leq N, \tau \in[0,1]$, and $h \in W_{p}^{s+1}(\mathbb{R})$, Lemma 2.1 yields

$$
\left\|\pi_{j}^{\varepsilon} \mathbb{B}(\tau f)\left[h^{\prime}\right]\right\|_{W_{p}^{s-2}} \leq\left\|\pi_{j}^{\varepsilon} \mathbb{B}(\tau f)\left[h^{\prime}\right]\right\|_{p} \leq C\left\|h^{\prime}\right\|_{p} \leq C\|h\|_{W_{p}^{s+1-\rho}},
$$

while, using some elementary arguments, we get

$$
\begin{aligned}
\left\|\pi_{j}^{\varepsilon}(\kappa(f)[h])^{\prime}\right\|_{W_{p}^{s-2}} & \leq\left\|\pi_{j}^{\varepsilon} \kappa(f)[h]\right\|_{W_{p}^{s-1}}+K\|\kappa(f)[h]\|_{p} \\
& \leq C_{0}\left\|\pi_{j}^{\varepsilon} h\right\|_{W_{p}^{s+1}}+K\|h\|_{W_{p}^{s+1-\rho}}
\end{aligned}
$$

Below we take advantage of (34) when considering the leading order term $\mathbb{B}(\tau f)\left[(\kappa(\tau f)[h])^{\prime}\right]$ of $\Phi(\tau f)[h]$. Let $C_{1}:=C_{0} k \sigma / 2 \pi \mu$.

Step 1: The case $|j| \leq N-1$. Given $|j| \leq N-1$, we infer from Lemma 4.2 and (34) that

$$
\begin{aligned}
& \left\|\pi_{j}^{\varepsilon} B_{0,1}^{0}(\tau f)\left[(\kappa(\tau f)[h])^{\prime}\right]-\frac{1}{1+\tau^{2} f^{\prime 2}\left(x_{j}^{\varepsilon}\right)} B_{0,0}\left[\pi_{j}^{\varepsilon}(\kappa(\tau f)[h])^{\prime}\right]\right\|_{W_{p}^{s-2}} \\
& \leq \frac{v}{4 C_{1}}\left\|\pi_{j}^{\varepsilon}(\kappa(\tau f)[h])^{\prime}\right\|_{W_{p}^{s-2}}+K\left\|(\kappa(\tau f)[h])^{\prime}\right\|_{W_{p}^{s-2-\rho}} \\
& \leq \frac{v C_{0}}{4 C_{1}}\left\|\pi_{j}^{\varepsilon} h\right\|_{W_{p}^{s+1}}+K\|h\|_{W_{p}^{s+1-\rho}}
\end{aligned}
$$

provided that $\varepsilon$ is sufficiently small. Besides, we have

$$
\left\|\pi_{j}^{\varepsilon} \tau f^{\prime} B_{1,1}^{0}(\tau f)\left[(\kappa(\tau f)[h])^{\prime}\right]-\frac{\tau^{2} f^{\prime 2}\left(x_{j}^{\varepsilon}\right)}{1+\tau^{2} f^{\prime 2}\left(x_{j}^{\varepsilon}\right)} B_{0,0}\left[\pi_{j}^{\varepsilon}(\kappa(\tau f)[h])^{\prime}\right]\right\|_{W_{p}^{s-2}} \leq T_{1}+T_{2}+T_{3},
$$

where

$$
\begin{aligned}
& T_{1}:=\left\|\chi_{j}^{\varepsilon}\left(f^{\prime}-f^{\prime}\left(x_{j}^{\varepsilon}\right)\right) B_{1,1}^{0}(\tau f)\left[\pi_{j}^{\varepsilon}(\kappa(\tau f)[h])^{\prime}\right]\right\|_{W_{p}^{s-2}}, \\
& T_{2}:=\left\|\chi_{j}^{\varepsilon}\left(f^{\prime}-f^{\prime}\left(x_{j}^{\varepsilon}\right)\right)\left(\pi_{j}^{\varepsilon} B_{1,1}^{0}(\tau f)\left[(\kappa(\tau f)[h])^{\prime}\right]-B_{1,1}^{0}(\tau f)\left[\pi_{j}^{\varepsilon}(\kappa(\tau f)[h])^{\prime}\right]\right)\right\|_{W_{p}^{s-2}}, \\
& T_{3}:=\left\|f^{\prime}\right\|_{\infty}\left\|\pi_{j}^{\varepsilon} B_{1,1}^{0}(\tau f)\left[(\kappa(\tau f)[h])^{\prime}\right]-\frac{\tau f^{\prime}\left(x_{j}^{\varepsilon}\right)}{1+\tau^{2} f^{\prime 2}\left(x_{j}^{\varepsilon}\right)} B_{0,0}\left[\pi_{j}^{\varepsilon}(\kappa(\tau f)[h])^{\prime}\right]\right\|_{W_{p}^{s-2}} .
\end{aligned}
$$

For $\varepsilon$ sufficiently small to guarantee that 


$$
\left\|\chi_{j}^{\varepsilon}\left(f^{\prime}-f^{\prime}\left(x_{j}^{\varepsilon}\right)\right)\right\|_{\infty}<\frac{v}{40 C_{1}}\left(\max _{\tau \in[0,1]}\left\|B_{1,1}^{0}(\tau f)\right\|_{\mathcal{L}\left(W_{p}^{s-2}(\mathbb{R})\right)}\right)^{-1},
$$

it follows from (7) (with $r=s-1$ ), Lemma 2.5, and (34) (if $\chi_{j}^{\varepsilon}\left(f^{\prime}-f^{\prime}\left(x_{j}^{\varepsilon}\right)\right.$ ) is not identically zero, otherwise the estimate is trivial) that

$$
T_{1} \leq \frac{\nu C_{0}}{8 C_{1}}\left\|\pi_{j}^{\varepsilon} h\right\|_{W_{p}^{s+1}}+K\|h\|_{W_{p}^{s+1-\rho}}
$$

As $\chi_{j}^{\varepsilon}\left(f^{\prime}-f^{\prime}\left(x_{j}^{\varepsilon}\right)\right) \in W_{p}^{s-1}(\mathbb{R})$ is a pointwise multiplier for $W_{p}^{s-2}(\mathbb{R})$, cf. (8), Lemma 4.1 yields

$$
T_{2} \leq K\left\|(\kappa(\tau f)[h])^{\prime}\right\|_{W_{p}^{s-2-\rho}} \leq K\|h\|_{W_{p}^{s+1-\rho}}
$$

Finally, if $\varepsilon$ is sufficiently small, we may argue as in the derivation of (35) to get

$$
T_{3} \leq \frac{v C_{0}}{8 C_{1}}\left\|\pi_{j}^{\varepsilon} h\right\|_{W_{p}^{s+1}}+K\|h\|_{W_{p}^{s+1-\rho}}
$$

Gathering (36)-(38), we conclude that

$$
\begin{aligned}
& \left\|\pi_{j}^{\varepsilon} \tau f^{\prime} B_{1,1}^{0}(\tau f)\left[(\kappa(\tau f)[h])^{\prime}\right]-\frac{\tau^{2} f^{\prime 2}\left(x_{j}^{\varepsilon}\right)}{1+\tau^{2} f^{\prime 2}\left(x_{j}^{\varepsilon}\right)} B_{0,0}\left[\pi_{j}^{\varepsilon}(\kappa(\tau f)[h])^{\prime}\right]\right\|_{W_{p}^{s-2}} \\
& \leq \frac{v C_{0}}{4 C_{1}}\left\|\pi_{j}^{\varepsilon} h\right\|_{W_{p}^{s+1}}+K\|h\|_{W_{p}^{s+1-\rho}} .
\end{aligned}
$$

We now combine (33), (35), and (39) and obtain that

$$
\left\|\pi_{j}^{\varepsilon} \Phi(\tau f)[h]-\frac{k \sigma}{2 \pi \mu} B_{0,0}\left[\pi_{j}^{\varepsilon}(\kappa(\tau f)[h])^{\prime}\right]\right\|_{W_{p}^{s-2}} \leq \frac{v}{2}\left\|\pi_{j}^{\varepsilon} h\right\|_{W_{p}^{s+1}}+K\|h\|_{W_{p}^{s+1-\rho}} .
$$

As a final step we show that, if $\varepsilon$ sufficiently small, then

$$
\left\|B_{0,0}\left[\pi_{j}^{\varepsilon}(\kappa(\tau f)[h])^{\prime}\right]-\frac{B_{0,0}\left[\left(\pi_{j}^{\varepsilon} h\right)^{\prime \prime \prime}\right]}{\left(1+\tau^{2} f^{\prime 2}\left(x_{j}^{\varepsilon}\right)\right)^{3 / 2}}\right\|_{W_{p}^{s-2}} \leq \frac{\nu C_{0}}{2 C_{1}}\left\|\pi_{j}^{\varepsilon} h\right\|_{W_{p}^{s+1}}+K\|h\|_{W_{p}^{s+1-\rho}}
$$

for all $h \in W_{p}^{s+1}(\mathbb{R}), \tau \in[0,1]$, and $|j| \leq N-1$. To start, we note that

$$
B_{0,0}\left[\pi_{j}^{\varepsilon}(\kappa(\tau f)[h])^{\prime}\right]=B_{0,0}\left[\left(\pi_{j}^{\varepsilon} \kappa(\tau f)[h]\right)^{\prime}\right]-B_{0,0}\left[\left(\pi_{j}^{\varepsilon}\right)^{\prime} \kappa(\tau f)[h]\right],
$$

and Lemma 2.5 yields for $-N+1 \leq j \leq N$ that 


$$
\left\|B_{0,0}\left[\left(\pi_{j}^{\varepsilon}\right)^{\prime} \kappa(\tau f)[h]\right]\right\|_{W_{p}^{s-2}} \leq K\|h\|_{W_{p}^{s+1-\rho} .}
$$

Moreover, letting $C_{2}:=\left\|B_{0,0}\right\|_{\mathcal{L}\left(W_{p}^{s-2}(\mathbb{R})\right)}$, the algebra property of $W_{p}^{s-1}(\mathbb{R})$ leads us to

$$
\begin{aligned}
& \left\|B_{0,0}\left[\left(\pi_{j}^{\varepsilon} \kappa(\tau f)[h]\right)^{\prime}\right]-\frac{B_{0,0}\left[\left(\pi_{j}^{\varepsilon} h\right)^{\prime \prime \prime}\right]}{\left(1+\tau^{2} f^{\prime 2}\left(x_{j}^{\varepsilon}\right)\right)^{3 / 2}}\right\|_{W_{p}^{s-2}} \\
& \left.\leq C_{2}\left\|\frac{\pi_{j}^{\varepsilon} h^{\prime \prime}}{\left(1+\tau^{2} f^{\prime 2}\right)^{3 / 2}}-\frac{\left(\pi_{j}^{\varepsilon} h\right)^{\prime \prime}}{\left(1+\tau^{2} f^{\prime 2}\left(x_{j}^{\varepsilon}\right)\right)^{3 / 2}}\right\|_{W_{p}^{s-1}}\right)^{\leq C_{2}}\left\|\left(\frac{1}{\left(1+\tau^{2} f^{\prime 2}\right)^{3 / 2}}-\frac{1}{\left(1+\tau^{2} f^{\prime 2}\left(x_{j}^{\varepsilon}\right)\right)^{3 / 2}}\right)^{\left(\pi_{j}^{\varepsilon} h\right)^{\prime \prime}}\right\|_{W_{p}^{s-1}}+K\|h\|_{W_{p}^{s}} .
\end{aligned}
$$

Using (5) together with the identity $\chi_{j}^{\varepsilon} \pi_{j}^{\varepsilon}=\pi_{j}^{\varepsilon}$, for $\varepsilon$ sufficiently small we get

$$
\begin{aligned}
& \left\|\left(\frac{1}{\left(1+\tau^{2} f^{\prime 2}\right)^{3 / 2}}-\frac{1}{\left(1+\tau^{2} f^{\prime 2}\left(x_{j}^{\varepsilon}\right)\right)^{3 / 2}}\right)\left(\pi_{j}^{\varepsilon} h\right)^{\prime \prime}\right\|_{W_{p}^{s-1}} \\
& \leq 2\left\|\chi_{j}^{\varepsilon}\left(\frac{1}{\left(1+\tau^{2} f^{\prime 2}\right)^{3 / 2}}-\frac{1}{\left(1+\tau^{2} f^{\prime 2}\left(x_{j}^{\varepsilon}\right)\right)^{3 / 2}}\right)\right\|_{\infty}\left\|\pi_{j}^{\varepsilon} h\right\|_{W_{p}^{s+1}}+K\|h\|_{W_{p}^{s+1-\rho}} \\
& \leq \frac{\nu C_{0}}{2 C_{1} C_{2}}\left\|\pi_{j}^{\varepsilon} h\right\|_{W_{p}^{s+1}}+K\|h\|_{W_{p}^{s+1-\rho}}
\end{aligned}
$$

Gathering (42)-(44), we conclude that (41) holds true. The desired estimate (32) follows now, for $|j| \leq N-1$, by combining (40) and (41).

Step 2: The case $j=N$. Similarly to (35), we obtain from Lemma 4.5 and (34) that

$$
\begin{aligned}
& \left\|\pi_{N}^{\varepsilon} B_{0,1}^{0}(\tau f)\left[(\kappa(\tau f)[h])^{\prime}\right]-B_{0,0}\left[\pi_{N}^{\varepsilon}(\kappa(\tau f)[h])^{\prime}\right]\right\|_{W_{p}^{s-2}} \\
& \leq \frac{\nu C_{0}}{4 C_{1}}\left\|\pi_{N}^{\varepsilon} h\right\|_{W_{p}^{s+1}}+K\|h\|_{W_{p}^{s+1-\rho}}
\end{aligned}
$$

for all $h \in W_{p}^{s+1}(\mathbb{R})$ and $\tau \in[0,1]$, provided that $\varepsilon$ is sufficiently small. Moreover,

$$
\left\|\pi_{N}^{\varepsilon} \tau f^{\prime} B_{1,1}^{0}(\tau f)\left[(\kappa(\tau f)[h])^{\prime}\right]\right\|_{W_{p}^{s-2}} \leq T_{a}+T_{b},
$$

where 


$$
\begin{aligned}
T_{a} & :=\left\|\chi_{N}^{\varepsilon} f^{\prime} B_{1,1}^{0}(\tau f)\left[\pi_{N}^{\varepsilon}(\kappa(\tau f)[h])^{\prime}\right]\right\|_{W_{p}^{s-2}}, \\
T_{b} & :=\left\|\chi_{N}^{\varepsilon} f^{\prime}\left(\pi_{N}^{\varepsilon} B_{1,1}^{0}(\tau f)\left[(\kappa(\tau f)[h])^{\prime}\right]-B_{1,1}^{0}(\tau f)\left[\pi_{N}^{\varepsilon}(\kappa(\tau f)[h])^{\prime}\right]\right)\right\|_{W_{p}^{s-2}} .
\end{aligned}
$$

Since $\chi_{N}^{\varepsilon} f^{\prime} \in W_{p}^{s-1}(\mathbb{R})$ is a pointwise multiplier for $W_{p}^{s-2}(\mathbb{R})$, cf. (8), Lemma 4.1 yields

$$
T_{b} \leq K\left\|(\kappa(\tau f)[h])^{\prime}\right\|_{W_{p}^{s-2-\rho}} \leq K\|h\|_{W_{p}^{s+1-\rho}} .
$$

Because $f^{\prime} \in W_{p}^{s-1}(\mathbb{R})$ vanishes at infinity, for $\varepsilon$ sufficiently small to ensure that

$$
\left\|\chi_{N}^{\varepsilon} f^{\prime}\right\|_{\infty}<\frac{v}{20 C_{1}}\left(\max _{\tau \in[0,1]}\left\|B_{1,1}^{0}(\tau f)\right\|_{\mathcal{L}\left(W_{p}^{s-2}(\mathbb{R})\right)}\right)^{-1},
$$

it follows from (7) (with $r=s-1$ ), Lemma 2.5, and (34) (if $\chi_{N}^{\varepsilon} f^{\prime}$ is not identically zero, otherwise the estimate is trivial) that

$$
T_{a} \leq \frac{v C_{0}}{4 C_{1}}\left\|\pi_{N}^{\varepsilon} h\right\|_{W_{p}^{s+1}}+K\|h\|_{W_{p}^{s+1-\rho}}
$$

Gathering (33) and (45)-(47), we have shown that if $\varepsilon$ is sufficiently small, then

$$
\left\|\pi_{N}^{\varepsilon} \Phi(\tau f)[h]-\frac{k \sigma}{2 \pi \mu} B_{0,0}\left[\pi_{N}^{\varepsilon}(\kappa(\tau f)[h])^{\prime}\right]\right\|_{W_{p}^{s-2}} \leq \frac{v}{2}\left\|\pi_{N}^{\varepsilon} h\right\|_{W_{p}^{s+1}}+K\|h\|_{W_{p}^{s+1-\rho}}
$$

for all $h \in W_{p}^{s+1}(\mathbb{R})$ and $\tau \in[0,1]$. It remains to show that for $\varepsilon$ sufficiently small

$$
\left\|B_{0,0}\left[\pi_{N}^{\varepsilon}(\kappa(\tau f)[h])^{\prime}\right]-B_{0,0}\left[\left(\pi_{N}^{\varepsilon} h\right)^{\prime \prime \prime}\right]\right\|_{W_{p}^{s-2}} \leq \frac{v C_{0}}{2 C_{1}}\left\|\pi_{N}^{\varepsilon} h\right\|_{W_{p}^{s+1}}+K\|h\|_{W_{p}^{s+1-\rho}}
$$

for all $h \in W_{p}^{s+1}(\mathbb{R})$ and $\tau \in[0,1]$. Arguing as in the first step (see (43)), we find in view of (42) that

$$
\begin{aligned}
& \left\|B_{0,0}\left[\pi_{N}^{\varepsilon}(\kappa(\tau f)[h])^{\prime}\right]-B_{0,0}\left[\left(\pi_{N}^{\varepsilon} h\right)^{\prime \prime \prime}\right]\right\|_{W_{p}^{s-2}} \\
& \leq C_{2}\left\|\left(\frac{1}{\left(\left(1+\tau^{2} f^{\prime 2}\right)^{3 / 2}\right.}-1\right)\left(\pi_{N}^{\varepsilon} h\right)^{\prime \prime}\right\|_{W_{p}^{s-1}}+K\|h\|_{W_{p}^{s+1-\rho}} .
\end{aligned}
$$

Using (5) and the fact that $f^{\prime}$ vanishes at infinity, for $\varepsilon$ sufficiently small, we obtain 


$$
\begin{aligned}
& C_{2}\left\|\left(\frac{1}{\left(\left(1+\tau^{2} f^{\prime 2}\right)^{3 / 2}\right.}-1\right)\left(\pi_{N}^{\varepsilon} h\right)^{\prime \prime}\right\|_{W_{p}^{s-1}} \\
& \leq 2 C_{2}\left\|\chi_{N}^{\varepsilon}\left(1-\left(1+\tau^{2} f^{\prime 2}\right)^{3 / 2}\right)\right\|_{\infty}\left\|\pi_{N}^{\varepsilon} h\right\|_{W_{p}^{s+1}}+K\|h\|_{W_{p}^{s+1-\rho}} \\
& \leq \frac{\nu C_{0}}{2 C_{1}}\left\|\pi_{N}^{\varepsilon} h\right\|_{W_{p}^{s+1}}+K\|h\|_{W_{p}^{s+1-\rho}} .
\end{aligned}
$$

This proves (49). The claim (32) for $j=N$ follows now directly from (48) and (49).

We now consider a class of Fourier multipliers related to the multipliers from Theorem 3.3.

Lemma 3.4 Let $\eta \in(0,1)$. Given $\alpha \in[\eta, 1 / \eta]$, let

$$
\mathbb{A}_{\alpha}:=-\alpha\left(\frac{d^{4}}{d x^{4}}\right)^{3 / 4}
$$

Then, there exits a constant $\kappa_{0}=\kappa_{0}(\eta) \geq 1$ such that

$$
\text { - } \quad \lambda-\mathbb{A}_{\alpha} \in \operatorname{Isom}\left(W_{p}^{s+1}(\mathbb{R}), W_{p}^{s-2}(\mathbb{R})\right) \quad \forall \operatorname{Re} \lambda \geq 1,
$$

- $\kappa_{0}\left\|\left(\lambda-\mathbb{A}_{\alpha}\right)[h]\right\|_{W_{p}^{s-2}} \geq|\lambda| \cdot\|h\|_{W_{p}^{s-2}}+\|h\|_{W_{p}^{s+1}} \quad \forall h \in W_{p}^{s+1}(\mathbb{R}), \operatorname{Re} \lambda \geq 1$.

Proof We first consider the realizations

$$
\mathbb{A}_{\alpha} \in \mathcal{L}\left(W_{p}^{2}(\mathbb{R}), W_{p}^{-1}(\mathbb{R})\right) \quad \text { and } \quad \mathbb{A}_{\alpha} \in \mathcal{L}\left(W_{p}^{3}(\mathbb{R}), L_{p}(\mathbb{R})\right)
$$

Since $W_{p}^{k}(\mathbb{R})=H_{p}^{k}(\mathbb{R}), k \in \mathbb{Z}$, Mikhlin's multiplier theorem, cf. e.g. [1, Theorem 4.23], shows that the properties (50)-(51) (in the appropriate spaces) are valid for these realizations. Then, using the interpolation property (3), we obtain that (50)(51) hold true.

The next result provides the generator property announced in (30).

Theorem 3.5 Given $p \in(1,2], s \in(1+1 / p, 2)$, and $f \in W_{p}^{s}(\mathbb{R})$ we have

$$
-\partial \Phi(f) \in \mathcal{H}\left(W_{p}^{s+1}(\mathbb{R}), W_{p}^{s-2}(\mathbb{R})\right) .
$$

Proof Fix $\rho \in(0, \min \{(s-1-1 / p) / 2), 2-s\})$. Since $f^{\prime} \in W_{p}^{s-1}(\mathbb{R})$ is bounded, there exists a constant $\eta>0$ with the property that the function $\alpha_{\tau}, \tau \in[0,1]$, from Theorem 3.3 satisfies $\eta \leq\left|\alpha_{\tau}\right| \leq 1 / \eta$ for all $\tau \in[0,1]$. Hence, regardless of $\varepsilon>0$, the operators $A_{j, \tau}$, with $-N+1 \leq j \leq N$ and $\tau \in[0,1]$, defined in Theorem 3.3 satisfy (50)-(51) with a constant $\kappa_{0} \geq 1$. 
We set $v:=\left(2 \kappa_{0}\right)^{-1}$. Theorem 3.3 provides an $\varepsilon \in(0,1)$, a $\varepsilon$-localization family, a constant $K=K(\varepsilon)>0$, and bounded operators $\mathbb{A}_{j, \tau} \in \mathcal{L}\left(W_{p}^{s+1}(\mathbb{R}), W_{p}^{s-2}(\mathbb{R})\right)$ ,$-N+1 \leq j \leq N$ and $\tau \in[0,1]$, such that

$$
2 \kappa_{0}\left\|\pi_{j}^{\varepsilon} \Phi(\tau f)[h]-\mathbb{A}_{j, \tau}\left[\pi_{j}^{\varepsilon} h\right]\right\|_{W_{p}^{s-2}} \leq\left\|\pi_{j}^{\varepsilon} h\right\|_{W_{p}^{s+1}}+2 \kappa_{0} K\|h\|_{W_{p}^{s+1-\rho}}
$$

for all $-N+1 \leq j \leq N, \tau \in[0,1]$, and $h \in W_{p}^{s+1}(\mathbb{R})$. Besides, Lemma 3.4 yields

$$
2 \kappa_{0}\left\|\left(\lambda-\mathbb{A}_{j, \tau}\right)\left[\pi_{j}^{\varepsilon} h\right]\right\|_{W_{p}^{s-2}} \geq 2|\lambda| \cdot\left\|\pi_{j}^{\varepsilon} h\right\|_{W_{p}^{s-2}}+2\left\|\pi_{j}^{\varepsilon} h\right\|_{W_{p}^{s+1}}
$$

for all $-N+1 \leq j \leq N, \tau \in[0,1]$, Re $\lambda \geq 1$, and $h \in W_{p}^{s+1}(\mathbb{R})$. The latter inequalities imply

$$
\begin{aligned}
2 \kappa_{0}\left\|\pi_{j}^{\varepsilon}(\lambda-\Phi(\tau f))[h]\right\|_{W_{p}^{s-2}} \geq & 2 \kappa_{0}\left\|\left(\lambda-\mathbb{A}_{j, \tau}\right)\left[\pi_{j}^{\varepsilon} h\right]\right\|_{W_{p}^{s-2}} \\
& -2 \kappa_{0}\left\|\pi_{j}^{\varepsilon} \Phi(\tau f)[h]-\mathbb{A}_{j, \tau}\left[\pi_{j}^{\varepsilon} h\right]\right\|_{W_{p}^{s-2}} \\
\geq & 2|\lambda| \cdot\left\|\pi_{j}^{\varepsilon} h\right\|_{W_{p}^{s-2}}+\left\|\pi_{j}^{\varepsilon} h\right\|_{W_{p}^{s+1}}-2 \kappa_{0} K\|f\|_{W_{p}^{s-1-\rho}}
\end{aligned}
$$

Summing up over $j$ and using Lemma 3.2, the interpolation property (3), and Young's inequality we find constants $\kappa=\kappa(f) \geq 1$ and $\omega=\omega(f)>0$ such that

$$
\kappa\|(\lambda-\Phi(\tau f))[h]\|_{W_{p}^{s-2}} \geq|\lambda| \cdot\|h\|_{W_{p}^{s-2}}+\|h\|_{W_{p}^{s+1}}
$$

for all $\tau \in[0,1]$, Re $\lambda \geq \omega$, and $h \in W_{p}^{s+1}(\mathbb{R})$.

Recalling (31) and arguing as in Lemma 3.4 we may choose $\omega$ sufficiently large to guarantee that $\omega-\Phi(0) \in \operatorname{Isom}\left(W_{p}^{s+1}(\mathbb{R}), W_{p}^{s-2}(\mathbb{R})\right)$. The method of continuity together with (52) consequently yields

$$
\omega-\Phi(f) \in \operatorname{Isom}\left(W_{p}^{s+1}(\mathbb{R}), W_{p}^{s-2}(\mathbb{R})\right) .
$$

The relations (52) (with $\tau=1$ ) and (53) imply the desired claim, cf. [7, Chapter I].

We next present the proof of the main result. The arguments rely to a large extent on the theory of quasilinear parabolic problems presented in $[3,4,6]$ (see also [28]). Besides, in order to obtain the parabolic smoothing properties, we additionally employ a parameter trick which was successfully applied also to other problems, cf., e.g., [9, 11, 18, 32].

Proof of Theorem 1.1 Fix

$$
1+\frac{1}{p}<\bar{s}<s<2 \quad \text { and } \quad 0<\beta:=\frac{2}{3}<\alpha:=\frac{s-\bar{s}+2}{3}<1 .
$$


We further set $E_{1}:=W_{p}^{\bar{s}+1}(\mathbb{R}), E_{0}:=W_{p}^{\bar{s}-2}(\mathbb{R})$, and $E_{\theta}:=\left(E_{0}, E_{1}\right)_{\theta, p}$, with $\theta \in\{\alpha, \beta\}$. Recalling the interpolation property (3), we have $E_{\alpha}=W_{p}^{s}(\mathbb{R})$ and $E_{\beta}=W_{p}^{\bar{s}}(\mathbb{R})$. In view of (29) and (30) (with $s=\bar{s}$ ), we then get

$$
-\Phi \in \mathrm{C}^{\infty}\left(E_{\beta}, \mathcal{H}\left(E_{1}, E_{0}\right)\right)
$$

and the assumptions of [28, Theorem 1.1] are satisfied in the context of the Muskat problem (27). Applying [28, Theorem 1.1], we may conclude that (27) has for each $f_{0} \in W_{p}^{s}(\mathbb{R})$ a unique maximal classical solution $f=f\left(\cdot ; f_{0}\right)$ such that

$$
f \in \mathrm{C}\left(\left[0, T^{+}\right), W_{p}^{s}(\mathbb{R})\right) \cap \mathrm{C}\left(\left(0, T^{+}\right), W_{p}^{\bar{s}+1}(\mathbb{R})\right) \cap \mathrm{C}^{1}\left(\left(0, T^{+}\right), W_{p}^{\bar{s}-2}(\mathbb{R})\right) .
$$

Moreover, if the solution belongs to the set

$$
\bigcup_{\eta \in(0,1)} \mathrm{C}^{\eta}\left(\left[0, T^{+}\right), W_{p}^{\bar{s}}(\mathbb{R})\right),
$$

then it is also unique, cf. [28, Remark 1.2 (ii)]. We now prove that each solution to (27) that satisfies (54) belongs to $\mathrm{C}^{\eta}\left(\left[0, T^{+}\right), W_{p}^{\bar{s}}(\mathbb{R})\right)$ with $\eta=(s-\bar{s}) /(3+s-\bar{s})$. Indeed, since $f \in \mathrm{C}\left(\left[0, T^{+}\right), W_{p}^{s}(\mathbb{R})\right)$, we infer from Lemma 3.6 that

$$
\sup _{t \in(0, T]}\left\|\frac{d f}{d t}(t)\right\|_{W_{p}^{\bar{s}-3}(\mathbb{R})}<\infty
$$

for each $\quad T \in\left(0, T^{+}\right)$. Since for $\theta:=3 /(s-\bar{s}+3)$ we have $\left(W_{p}^{\bar{s}-3}(\mathbb{R}), W_{p}^{s}(\mathbb{R})\right)_{\theta, p}=W_{p}^{\bar{s}}(\mathbb{R})$, cf. (3), (54) and the latter estimate now yield

$$
\left\|f\left(t_{1}\right)-f\left(t_{2}\right)\right\|_{W_{p}^{\bar{s}}} \leq C\left\|f\left(t_{1}\right)-f\left(t_{2}\right)\right\|_{W_{p}^{s-3}}^{1-\theta} \leq C\left|t_{1}-t_{2}\right|^{\theta(s-\bar{s}) / 3}, \quad t_{1}, t_{2} \in[0, T] .
$$

Therewith the existence and uniqueness claim is proven. Moreover, the assertion (i) follows from the abstract theory (see the proof of [28, Theorem 1.1]).

The parabolic smoothing property established at (ii) can be shown by arguing as in the more restrictive case considered in [25, Theorem 1.3].

Finally, in order to prove (iii), we assume that $f=f\left(\cdot ; f_{0}\right):\left[0, T^{+}\right) \rightarrow W_{p}^{s}(\mathbb{R})$ is a maximal classical solution with $T^{+}<\infty$ and that

$$
\sup _{t \in\left[0, T^{+}\right)}\|f(t)\|_{W_{p}^{s}(\mathbb{R})}<\infty .
$$

Using again Lemma 3.6 and arguing as above, we conclude that $f:\left[0, T^{+}\right) \rightarrow W_{p}^{\bar{s}}(\mathbb{R})$ is uniformly continuous. Let now

$$
1+\frac{1}{p}<\tilde{s}<\bar{s}<2 \quad \text { and } \quad 0<\beta:=\frac{2}{3}<\alpha^{\prime}:=\frac{\bar{s}-\tilde{s}+2}{3}<1 .
$$


Choosing $\quad F_{1}:=W_{p}^{\widetilde{s}+1}(\mathbb{R}), \quad F_{0}:=W_{p}^{\widetilde{s}-2}(\mathbb{R}), \quad$ and $\quad$ setting $\quad F_{\theta}:=\left(F_{0}, F_{1}\right)_{\theta, p}$, $\theta \in\left\{\alpha^{\prime}, \beta\right\}$, we have that $F_{\alpha}=W_{p}^{\bar{s}}(\mathbb{R})$ and $F_{\beta}=W_{p}^{\widetilde{s}}(\mathbb{R})$. Moreover (29) and (30) (with $s=\widetilde{s}$ ), yield

$$
-\Phi \in \mathrm{C}^{\infty}\left(F_{\beta}, \mathcal{H}\left(F_{1}, F_{0}\right)\right)
$$

Thus, we may apply again [28, Theorem 1.1] (iv) ( $\alpha$ ) to (27) and conclude that $f$ can be extended to an interval $\left[0, \widetilde{T}^{+}\right)$with $\widetilde{T}^{+}>T^{+}$and such that

$$
f \in \mathrm{C}\left(\left[0, \widetilde{T}^{+}\right), W_{p}^{\bar{s}}(\mathbb{R})\right) \cap \mathrm{C}\left(\left(0, \widetilde{T}^{+}\right), W_{p}^{\widetilde{s}+1}(\mathbb{R})\right) \cap \mathrm{C}^{1}\left(\left(0, \widetilde{T}^{+}\right), W_{p}^{\widetilde{s}-2}(\mathbb{R})\right) .
$$

Moreover, by (ii) (with $(s, \bar{s})=(\bar{s}, \widetilde{s}))$ we also have $f \in C^{1}\left(\left(0, \widetilde{T}^{+}\right), W_{p}^{3}(\mathbb{R})\right)$, and this contradicts the maximality of $f$. This proves the claim (iii) and the argument is complete.

We finish the section by presenting a result used in the proof of Theorem 1.1.

Lemma 3.6 Given $M>0$, there exists a constant $C=C(M)$ such that

$$
\|\Phi(f)[f]\|_{W_{p}^{s-3}} \leq C
$$

for all $f \in W_{p}^{s+1}(\mathbb{R})$ with $\|f\|_{W_{p}^{s}} \leq M$.

Proof In this proof the constants denoted by $C$ depend only on $M$. Given $f \in W_{p}^{s+1}(\mathbb{R})$ with $\|f\|_{W_{p}^{s}} \leq M$, we have

$$
\left\|(\kappa(f)[f])^{\prime}\right\|_{W_{p}^{s-3}(\mathbb{R})}=\left\|\left(\frac{f^{\prime}}{\left(1+f^{\prime 2}\right)^{1 / 2}}\right)^{\prime \prime}\right\|_{W_{p}^{s-3}} \leq C\left\|\frac{f^{\prime}}{\left(1+f^{\prime 2}\right)^{1 / 2}}\right\|_{W_{p}^{s-1}} \leq C .
$$

Moreover, we infer from Lemma 2.1 that

$$
\left\|\mathbb{B}(f)\left[f^{\prime}\right]\right\|_{W_{p}^{s-3}} \leq\left\|\mathbb{B}(f)\left[f^{\prime}\right]\right\|_{p} \leq C
$$

and it remains to prove that

$$
\left\|\mathbb{B}(f)\left[(\kappa(f)[f])^{\prime}\right]\right\|_{W_{p}^{s-3}} \leq C .
$$

To this end we note that the $L_{2}$-adjoint $\mathbb{B}^{*}(f)$ of $\mathbb{B}(f)$ is identified by the relation

$$
\pi \mathbb{B}^{*}(f):=-\left(B_{0,1}(f)+B_{1,1}(f)\left[f, f^{\prime} \cdot\right]\right) .
$$

Therefore, given $\bar{\omega}, \psi \in \mathrm{C}_{0}^{\infty}(\mathbb{R})$, we have

$$
\begin{aligned}
\left|\langle\mathbb{B}(f)[\bar{\omega}] \mid \psi\rangle_{W_{p}^{s-3}(\mathbb{R}) \times W_{p^{\prime}}^{3-s}(\mathbb{R})}\right| & =\left|\left\langle\bar{\omega} \mid \mathbb{B}^{*}(f)[\psi]\right\rangle_{W_{p}^{s-3}(\mathbb{R}) \times W_{p^{\prime}}^{3-s}(\mathbb{R})}\right| \\
& \leq\|\bar{\omega}\|_{W_{p}^{s-3}}\left\|\mathbb{B}^{*}(f)[\psi]\right\|_{W_{p^{\prime}}^{3-s}}
\end{aligned}
$$


We next show that

$$
\left\|\mathbb{B}^{*}(f)[\psi]\right\|_{W_{p^{\prime}}^{3-s}} \leq C\|\psi\|_{W_{p^{\prime}}^{3-s}}
$$

for all $\psi \in W_{p^{\prime}}^{3-s}(\mathbb{R})$ and $f \in W_{p}^{s+1}(\mathbb{R})$ with $\|f\|_{W_{p}^{s}} \leq M$. Indeed, Lemma 2.1 (with $p=p^{\prime}$ ), yields

$$
\left\|\mathbb{B}^{*}(f)[\psi]\right\|_{p^{\prime}} \leq C\|\psi\|_{p^{\prime}}
$$

Additionally, we may argue as in the proof of [25, Lemma 3.5] to infer in view of Lemma 2.1 that $\mathbb{B}^{*}(f)[\psi] \in W_{p^{\prime}}^{1}(\mathbb{R})$ with

$$
\begin{aligned}
\pi\left(\mathbb{B}^{*}(f)[\psi]\right)^{\prime}(x) & =\pi \mathbb{B}^{*}(f)\left[\psi^{\prime}\right](x)-\mathrm{PV} \int_{\mathbb{R}} \partial_{x}\left(\frac{y+f^{\prime}(x-y) \delta_{[x, y]} f}{y^{2}+\left(\delta_{[x, y]} f\right)^{2}}\right) \psi(x-y) d y \\
& =\pi \mathbb{B}^{*}(f)\left[\psi^{\prime}\right](x)-\mathrm{PV} \int_{\mathbb{R}} \partial_{y}\left(\frac{\delta_{[x, y]} f \delta_{[x, y]} f^{\prime}}{y^{2}+\left(\delta_{[x, y]} f\right)^{2}}\right) \psi(x-y) d y \\
& =-\pi \mathbb{B}(f)\left[\psi^{\prime}\right](x) .
\end{aligned}
$$

Invoking Lemma 2.4 (with $r=2-s \in(0,1-1 / p)$ ), we get that

$$
\left\|B_{0,1}^{0}(f)\left[\psi^{\prime}\right]\right\|_{W_{p^{\prime}}^{2-s}}+\left\|B_{1,1}^{0}(f)\left[\psi^{\prime}\right]\right\|_{W_{p^{\prime}}^{2-s}} \leq C\|\psi\|_{W_{p^{\prime}}^{3-s}},
$$

and since $f^{\prime} \in W_{p}^{s-1}(\mathbb{R})$ is a pointwise multiplier for $W_{p^{\prime}}^{2-s}(\mathbb{R})$, cf. (8), we may conclude from (61) and (62) that

$$
\left\|\mathbb{B}^{*}(f)[\psi]\right\|_{W_{p^{\prime}}^{3-s}} \leq\left\|\mathbb{B}^{*}(f)[\psi]\right\|_{p^{\prime}}+\left\|\left(\mathbb{B}^{*}(f)[\psi]\right)^{\prime}\right\|_{W_{p^{\prime}}^{2-s}} \leq C\|\psi\|_{W_{p^{\prime}}^{3-s}}
$$

This proves (60). Combining (59) and (60), a standard density argument leads us to

$$
\|\mathbb{B}(f)[\bar{\omega}]\|_{W_{p}^{s-3}} \leq C\|\bar{\omega}\|_{W_{p}^{s-3}}, \quad \bar{\omega} \in W_{p}^{s-3}(\mathbb{R}),
$$

and (58) follows via (56). Recalling the definition (28) of $\Phi$, the bound (55) is a straightforward consequence of (57) and (58).

\section{Freezing the kernels of singular operators in Sobolev spaces with negative exponent}

In this section we establish some technical results which enable us to locally approximate the Fréchet derivative $\partial \Phi(\tau f), \tau \in[0,1]$ and $f \in W_{p}^{s}(\mathbb{R})$, by certain explicit Fourier multipliers, cf. Theorem 3.3. These results can be viewed as a generalization of the method of freezing the coefficients of elliptic differential operators. We extend this method to a particular class of singular integral operators, one of the main difficulties arising from the fact that the Sobolev space where the error is measured has negative exponent. As a first result we establish a commutator type estimate. 
Lemma 4.1 Let $p \in(1,2], s \in(1+1 / p, 2), \quad \rho \in(0,(s-1-1 / p) / 2), n, m \in \mathbb{N}$, $f \in W_{p}^{s}(\mathbb{R})$, and $\varphi \in C^{1}(\mathbb{R})$ with uniformly continuous derivative $\varphi^{\prime}$ be given. Then, there exists a constant $K=K\left(n, m, s, p, \rho,\|\varphi\|_{C^{1}},\|f\|_{W_{p}^{s}}\right)$ such that

$$
\left\|\varphi B_{n, m}^{0}(f)[\bar{\omega}]-B_{n, m}^{0}(f)[\varphi \bar{\omega}]\right\|_{W_{p}^{s-2}} \leq K\|\bar{\omega}\|_{W_{p}^{s-2-\rho}} \quad \text { for all } \quad \bar{\omega} \in W_{p}^{s-2}(\mathbb{R}) .
$$

Proof Let $\bar{\rho}:=(3-s) \rho /(1-\rho)$. We point out that $\bar{\rho} \in(\rho, s-1-1 / p)$ and that $\varphi \in \mathrm{C}^{1}(\mathbb{R})$ is a pointwise multiplier for $W_{p}^{s-2-\bar{\rho}}(\mathbb{R})$. Lemma 2.5 (with $s=s-\bar{\rho}$ ) then yields

$$
\left\|\varphi B_{n, m}^{0}(f)[\bar{\omega}]-B_{n, m}^{0}(f)[\varphi \bar{\omega}]\right\|_{W_{p}^{s-2-\bar{\rho}}} \leq K\|\bar{\omega}\|_{W_{p}^{s-2-\bar{\rho}},} \quad \bar{\omega} \in W_{p}^{s-2-\bar{\rho}}(\mathbb{R}) .
$$

Besides, in view of [2, Lemma 12], we also have

$$
\left\|\varphi B_{n, m}^{0}(f)[\bar{\omega}]-B_{n, m}^{0}(f)[\varphi \bar{\omega}]\right\|_{W_{p}^{1}} \leq K\|\bar{\omega}\|_{p}, \quad \bar{\omega} \in L_{p}(\mathbb{R}) .
$$

Noticing that $W_{p}^{(1-\rho)(s-2-\bar{\rho})}(\mathbb{R})=W_{p}^{s-2-\bar{\rho}}(\mathbb{R})$ and $W_{p}^{(1-\rho)(s-2-\bar{\rho})+\rho}(\mathbb{R})=W_{p}^{s-2}(\mathbb{R})$, the interpolation property (3) together with (64) and (65) leads us to the desired claim.

The first important result of this section is the following lemma.

Lemma 4.2 Let $n, m \in \mathbb{N}, \quad p \in(1,2], s \in(1+1 / p, 2), \quad \rho \in(0,(s-1-1 / p) / 2)$, $v \in(0, \infty)$, and $f \in W_{p}^{s}(\mathbb{R})$ be given. For sufficiently small $\varepsilon \in(0,1)$ there exists a constant $K$ that depends on $\varepsilon, n, m, s, p, \rho$, and $\|f\|_{W_{p}^{s}}$ such that

$$
\left\|\pi_{j}^{\varepsilon} B_{n, m}^{0}(f)[\bar{\omega}]-\frac{\left(f^{\prime}\left(x_{j}^{\varepsilon}\right)\right)^{n}}{\left[1+\left(f^{\prime}\left(x_{j}^{\varepsilon}\right)\right)^{2}\right]^{m}} B_{0,0}\left[\pi_{j}^{\varepsilon} \bar{\omega}\right]\right\|_{W_{p}^{s-2}} \leq v\left\|\pi_{j}^{\varepsilon} \bar{\omega}\right\|_{W_{p}^{s-2}}+K\|\bar{\omega}\|_{W_{p}^{s-2-\rho}}
$$

for all $|j| \leq N-1$ and $\bar{\omega} \in W_{p}^{s-2}(\mathbb{R})$.

Proof Taking advantage of the relation $\chi_{j}^{\varepsilon} \pi_{j}^{\varepsilon}=\pi_{j}^{\varepsilon}$, we have

$$
\pi_{j}^{\varepsilon} B_{n, m}^{0}(f)[\bar{\omega}]-\frac{\left(f^{\prime}\left(x_{j}^{\varepsilon}\right)\right)^{n}}{\left[1+\left(f^{\prime}\left(x_{j}^{\varepsilon}\right)\right)^{2}\right]^{m}} B_{0,0}\left[\pi_{j}^{\varepsilon} \bar{\omega}\right]=T_{a}+T_{b},
$$

where 


$$
\begin{aligned}
T_{a} & :=\frac{\left(f^{\prime}\left(x_{j}^{\varepsilon}\right)\right)^{n}}{\left[1+\left(f^{\prime}\left(x_{j}^{\varepsilon}\right)\right)^{2}\right]^{m}}\left(\chi_{j}^{\varepsilon} B_{0,0}\left[\pi_{j}^{\varepsilon} \bar{\omega}\right]-B_{0,0}\left[\chi_{j}^{\varepsilon}\left(\pi_{j}^{\varepsilon} \bar{\omega}\right)\right]\right) \\
& -\chi_{j}^{\varepsilon}\left(B_{n, m}^{0}(f)\left[\pi_{j}^{\varepsilon} \bar{\omega}\right]-\pi_{j}^{\varepsilon} B_{n, m}^{0}(f)[\bar{\omega}]\right), \\
T_{b} & :=\chi_{j}^{\varepsilon} B_{n, m}^{0}(f)\left[\pi_{j}^{\varepsilon} \bar{\omega}\right]-\frac{\left(f^{\prime}\left(x_{j}^{\varepsilon}\right)\right)^{n}}{\left[1+\left(f^{\prime}\left(x_{j}^{\varepsilon}\right)\right)^{2}\right]^{m}} \chi_{j}^{\varepsilon} B_{0,0}\left[\pi_{j}^{\varepsilon} \bar{\omega}\right] .
\end{aligned}
$$

Since $\pi_{j}^{\varepsilon}$ is a pointwise multiplier for $W_{p}^{s-2-\rho}(\mathbb{R})$ and $\chi_{j}^{\varepsilon}$ is a pointwise multiplier for $W_{p}^{s-2}(\mathbb{R})$, Lemma 4.1 leads to

$$
\left\|T_{a}\right\|_{W_{p}^{s-2}} \leq K\|\bar{\omega}\|_{W_{p}^{s-2-\rho}}
$$

It remains to estimate $T_{b}$. Using (14) and the relation $f^{\prime}\left(x_{j}^{\varepsilon}\right)=\delta_{[x, y]}\left(f^{\prime}\left(x_{j}^{\varepsilon}\right) \operatorname{id}_{\mathbb{R}}\right) / y$, we get

$$
\begin{aligned}
T_{b} & =\sum_{k=0}^{n-1}\left(f^{\prime}\left(x_{j}^{\varepsilon}\right)\right)^{n-k-1} \chi_{j}^{\varepsilon} B_{k+1, m}(f, \ldots, f)\left[f, \ldots, f, f-f^{\prime}\left(x_{j}^{\varepsilon}\right) \mathrm{id}_{\mathbb{R}}, \pi_{j}^{\varepsilon} \bar{\omega}\right] \\
& -\sum_{k=0}^{m-1} \frac{\left(f^{\prime}\left(x_{j}^{\varepsilon}\right)\right)^{n}}{\left[1+\left(f^{\prime}\left(x_{j}^{\varepsilon}\right)\right)^{2}\right]^{m-k}} \chi_{j}^{\varepsilon} B_{2, k+1}(f, \ldots, f)\left[f-f^{\prime}\left(x_{j}^{\varepsilon}\right) \mathrm{id}_{\mathbb{R}}, f+f^{\prime}\left(x_{j}^{\varepsilon}\right) \mathrm{id}_{\mathbb{R}}, \pi_{j}^{\varepsilon} \bar{\omega}\right] .
\end{aligned}
$$

If $\varepsilon$ is sufficiently small, Lemma 4.4 (with $\widetilde{s}=s-\rho$ ) implies that

$$
\left\|T_{b}\right\|_{W_{p}^{s-2}} \leq v\left\|\pi_{j}^{\varepsilon} \bar{\omega}\right\|_{W_{p}^{s-2}}+K\|\bar{\omega}\|_{W_{p}^{s-2-\rho}} .
$$

The estimate (66) follows from (67) and (68).

In Lemma 4.3 we gather some classical properties of mollifiers.

Lemma 4.3 Let $\eta_{\delta}(x):=\delta^{-1} \eta(x / \delta), x \in \mathbb{R}$ and $\delta>0$, where $\eta \in \mathrm{C}_{0}^{\infty}(\mathbb{R})$ is a nonnegative function with

$$
\int_{\mathbb{R}} \eta(x) d x=1
$$

Given $\delta>0$ and $f \in L_{p}(\mathbb{R})$, with $p \in[1, \infty)$, let $f_{\delta}:=f * \eta_{\delta}$. The following properties hold true.

(i) Given $r \geq 0$, it holds $\sup _{\delta>0}\left\|\left[f \mapsto f_{\delta}\right]\right\|_{\mathcal{L}\left(W_{p}^{r(\mathbb{R}))}\right.}=1$;

(ii) Given $r \geq 0$, there exists a constant $C>0$ such that 


$$
\left\|f_{\delta}\right\|_{W_{p}^{r}} \leq C \delta^{r^{\prime}-r}\|f\|_{W_{p}^{\prime}} \quad \text { for all } f \in W_{p}^{r^{\prime}}(\mathbb{R}), r^{\prime} \in[0, r], \delta \in(0,1] .
$$

(iii) There exists a constant $C>0$ such that

$$
\left\|f_{\delta}-f\right\|_{W_{p}^{\prime}} \leq C \delta^{r-r^{\prime}}\|f\|_{W_{p}^{r}} \quad \text { for all } f \in W_{p}^{r}(\mathbb{R}), 0 \leq r^{\prime} \leq r \leq 1, \delta>0 .
$$

Lemma 4.4 below provides the key estimate in the proof of Lemma 4.2.

Lemma 4.4 Let $n, m \in \mathbb{N}, \quad n \geq 1, \quad p \in(1,2], 1+1 / p<\tilde{s}<s<2, \quad v \in(0, \infty)$, and $f \in W_{p}^{s}(\mathbb{R})$ be given. For sufficiently small $\varepsilon \in(0,1)$ there exists a positive constant $K=K\left(\varepsilon, n, m, s, \widetilde{s},\|f\|_{W_{p}^{s}}\right)$ such that

$$
\left\|\chi_{j}^{\varepsilon} B_{n, m}(f, \ldots, f)\left[f, \ldots, f, f-f^{\prime}\left(x_{j}^{\varepsilon}\right) \operatorname{id}_{\mathbb{R}}, \pi_{j}^{\varepsilon} \bar{\omega}\right]\right\|_{W_{p}^{s-2}} \leq v\left\|\pi_{j}^{\varepsilon} \bar{\omega}\right\|_{W_{p}^{s-2}}+K\|\bar{\omega}\|_{W_{p}^{\widetilde{s}-2}}
$$

for all $|j| \leq N-1$ and $\bar{\omega} \in W_{p}^{s-2}(\mathbb{R})$.

Proof Given $\varphi, \bar{\omega} \in \mathrm{C}_{0}^{\infty}(\mathbb{R})$, it holds in view of $\chi_{j}^{\varepsilon} \pi_{j}^{\varepsilon}=\pi_{j}^{\varepsilon}$ and of the fact that the $L_{2}$ -adjoint of $B_{n, m}^{0}(f)$ is the operator $-B_{n, m}^{0}(f)$, that

$$
\begin{aligned}
& \left\langle\chi_{j}^{\varepsilon} B_{n, m}(f, \ldots, f)\left[f, \ldots, f, f-f^{\prime}\left(x_{j}^{\varepsilon}\right) \operatorname{id}_{\mathbb{R}}, \pi_{j}^{\varepsilon} \bar{\omega}\right] \mid \varphi\right\rangle_{W_{p}^{s-2}(\mathbb{R}) \times W_{p^{\prime}}^{2-s}(\mathbb{R})} \\
& =-\left\langle\pi_{j}^{\varepsilon} \bar{\omega} \mid \chi_{j}^{\varepsilon} B_{n, m}(f, \ldots, f)\left[f, \ldots, f, f-f^{\prime}\left(x_{j}^{\varepsilon}\right) \operatorname{id}_{\mathbb{R}}, \chi_{j}^{\varepsilon} \varphi\right]\right\rangle_{W_{p}^{s-2}(\mathbb{R}) \times W_{p^{\prime}}^{2-s}(\mathbb{R})} .
\end{aligned}
$$

Let $T_{j, \varepsilon}[\varphi]:=\chi_{j}^{\varepsilon} B_{n, m}(f, \ldots, f)\left[f, \ldots, f, f-f^{\prime}\left(x_{j}^{\varepsilon}\right) \mathrm{id}_{\mathbb{R}}, \chi_{j}^{\varepsilon} \varphi\right]$. We decompose below

$$
T_{j, \varepsilon}[\varphi]=T_{j, \varepsilon}^{1}[\varphi]+T_{j, \varepsilon}^{2}[\varphi],
$$

and we prove subsequently that, if $\varepsilon$ sufficiently small, then

$$
\left\|T_{j, \varepsilon}^{1}[\varphi]\right\|_{W_{p^{\prime}}^{2-s}} \leq \nu\|\varphi\|_{W_{p^{\prime}}^{2-s}} \quad \text { and } \quad\left\|T_{j, \varepsilon}^{2}[\varphi]\right\|_{W_{p^{\prime}}^{2-\tau^{\prime}}} \leq K\|\varphi\|_{W_{p^{\prime}}^{2-s}}
$$

for all $\varphi \in W_{p^{\prime}}^{2-s}(\mathbb{R})$. Having established (73), we get

$$
\begin{aligned}
\left|\left\langle\pi_{j}^{\varepsilon} \bar{\omega} \mid T_{j, \varepsilon}[\varphi]\right\rangle_{W_{p}^{s-2}(\mathbb{R}) \times W_{p^{\prime}}^{2-s}(\mathbb{R})}\right| & \leq\left\|\pi_{j}^{\varepsilon} \bar{\omega}\right\|_{W_{p}^{s-2}}\left\|T_{j, \varepsilon}^{1}[\varphi]\right\|_{W_{p^{\prime}}^{2-s}}+\left\|\pi_{j}^{\varepsilon} \bar{\omega}\right\|_{W_{p}^{\tilde{s}-2}}\left\|T_{j, \varepsilon}^{2}[\varphi]\right\|_{W_{p^{\prime}}^{2-\tau}} \\
& \leq\left(\nu\left\|\pi_{j}^{\varepsilon} \bar{\omega}\right\|_{W_{p}^{s-2}}+K\|\bar{\omega}\|_{W_{p}^{\tilde{s}-2}}\right)\|\varphi\|_{W_{p^{\prime}}^{2-s}}
\end{aligned}
$$

and the claim (71) follows via a standard density argument.

In order to define the terms in (72), let $\left\{\eta_{\delta}\right\}_{\delta>0}$ be a mollifier as in Lemma 4.3. We set 


$$
\begin{aligned}
& T_{j, \varepsilon}^{1}[\varphi]=\chi_{j}^{\varepsilon} B_{n, m}(f, \ldots, f)\left[f, \ldots, f, f-f^{\prime}\left(x_{j}^{\varepsilon}\right) \mathrm{id}_{\mathbb{R}}, \chi_{j}^{\varepsilon}\left(\varphi-\varphi_{\delta}\right)\right], \\
& T_{j, \varepsilon}^{2}[\varphi]=\chi_{j}^{\varepsilon} B_{n, m}^{0}(f)\left[\chi_{j}^{\varepsilon} \varphi_{\delta}\right]-f^{\prime}\left(x_{j}^{\varepsilon}\right) \chi_{j}^{\varepsilon} B_{n-1, m}^{0}(f)\left[\chi_{j}^{\varepsilon} \varphi_{\delta}\right],
\end{aligned}
$$

where $\delta=\delta(\varepsilon) \in(0,1]$ will be fixed later on and $\varphi_{\delta}:=\varphi * \eta_{\delta}$.

Since $2-\widetilde{s} \in[0,1-1 / p$ ), Lemma 2.4 (with $r=2-\widetilde{s}$ ) and the fact that $\chi_{j}^{\varepsilon} \varphi_{\delta} \in W_{p^{\prime}}^{2-\widetilde{s}}(\mathbb{R})$ yield $T_{j, \varepsilon}^{2}[\varphi] \in W_{p^{\prime}}^{2-\widetilde{s}}(\mathbb{R})$, and together with (69) we obtain the estimate

$$
\left\|T_{j, \varepsilon}^{2}[\varphi]\right\|_{W_{p^{\prime}}^{2-\widetilde{s}}} \leq K\left\|\chi_{j}^{\varepsilon} \varphi_{\delta}\right\|_{W_{p^{\prime}}^{2-\widetilde{s}}} \leq K \delta^{\widetilde{s}-s}\|\varphi\|_{W_{p^{\prime}}^{2-s}}
$$

It remains to estimate $T_{j, \varepsilon}^{1}[\varphi]$. Therefore, we first infer from Lemma 2.1 and (70) that

$$
\left\|T_{j, \varepsilon}^{1}[\varphi]\right\|_{p^{\prime}} \leq C\left\|\chi_{j}^{\varepsilon}\left(\varphi_{\delta}-\varphi\right)\right\|_{p^{\prime}} \leq C\left\|\varphi_{\delta}-\varphi\right\|_{p^{\prime}} \leq C \delta^{2-s}\|\varphi\|_{W_{p^{\prime}}^{2-s}}
$$

We now consider the seminorm $\left[T_{j, \varepsilon}^{1}[\varphi]\right]_{W_{p^{\prime}}^{2-s}}$. In view of (14) we write

$$
T_{j, \varepsilon}^{1}[\varphi]-\tau_{\xi} T_{j, \varepsilon}^{1}[\varphi]=T_{1}(\xi)+T_{2}(\xi)+T_{3}(\xi), \quad \xi \in \mathbb{R}
$$

where

$$
\begin{aligned}
T_{1}(\xi) & :=\left(\chi_{j}^{\varepsilon}-\tau_{\xi} \chi_{j}^{\varepsilon}\right) \tau_{\xi} B_{n, m}(f, \ldots, f)\left[f, \ldots, f, f-f^{\prime}\left(x_{j}^{\varepsilon}\right) \operatorname{id}_{\mathbb{R}}, \chi_{j}^{\varepsilon}\left(\varphi-\varphi_{\delta}\right)\right], \\
T_{2}(\xi) & :=\chi_{j}^{\varepsilon} B_{n, m}(f, \ldots, f)\left[f, \ldots, f, f-f^{\prime}\left(x_{j}^{\varepsilon}\right) \operatorname{id}_{\mathbb{R}}, \chi_{j}^{\varepsilon}\left(\varphi-\varphi_{\delta}\right)-\tau_{\xi}\left(\chi_{j}^{\varepsilon}\left(\varphi-\varphi_{\delta}\right)\right)\right], \\
T_{3}(\xi) & :=\chi_{j}^{\varepsilon} \sum_{j=1}^{n-1} B_{n, m}(f, \ldots, f)[\underbrace{\tau_{\xi} f, \ldots, \tau_{\xi}}_{(j-1)-\operatorname{times}} f, f-\tau_{\xi} f, f, \ldots, f, f-f^{\prime}\left(x_{j}^{\varepsilon}\right) \operatorname{id}_{\mathbb{R}}, \tau_{\xi}\left(\chi_{j}^{\varepsilon}\left(\varphi-\varphi_{\delta}\right)\right)] \\
& +\chi_{j}^{\varepsilon} B_{n, m}(f, \ldots, f)\left[\tau_{\xi} f, \ldots, \tau_{\xi} f, f-\tau_{\xi} f, \tau_{\xi}\left(\chi_{j}^{\varepsilon}\left(\varphi-\varphi_{\delta}\right)\right)\right] \\
& +\chi_{j}^{\varepsilon} \sum_{j=1}^{m} B_{n+2, m+1}^{j}\left[\tau_{\xi} f, \ldots, \tau_{\xi} f, \tau_{\xi} f-f^{\prime}\left(x_{j}^{\varepsilon}\right) \operatorname{id}_{\mathbb{R}}, \tau_{\xi} f+f, \tau_{\xi} f-f, \tau_{\xi}\left(\chi_{j}^{\varepsilon}\left(\varphi-\varphi_{\delta}\right)\right)\right],
\end{aligned}
$$

with

$$
B_{n+2, m+1}^{j}:=B_{n+2, m+1}(\underbrace{f, \ldots, f}_{j-\text { times }}, \tau_{\xi} f, \ldots, \tau_{\xi} f) .
$$

Lemma 2.1 (with $p=p^{\prime}$ ) together wit (70) yields

$$
\left\|T_{1}(\xi)\right\|_{p^{\prime}} \leq C\left\|\chi_{j}^{\varepsilon}-\tau_{\xi} \chi_{j}^{\varepsilon}\right\|_{\infty}\left\|\chi_{j}^{\varepsilon}\left(\varphi-\varphi_{\delta}\right)\right\|_{p^{\prime}} \leq C \delta^{2-s}\left\|\chi_{j}^{\varepsilon}-\tau_{\xi} \chi_{j}^{\varepsilon}\right\|_{W_{p^{\prime}}^{1}}\|\varphi\|_{W_{p^{\prime}}^{2-s}} .
$$

With regard to $T_{3}(\xi)$ we fix $\rho \in(s-\tilde{s}, \min \{2-\tilde{s}, 2(s-\tilde{s})\})$. Combining Lemma 2.2 (with $s=\widetilde{s}$ and $r=2-\widetilde{s}-\rho \in(0,1-1 / p)$ ) and (70) we get 


$$
\begin{aligned}
\left\|T_{3}(\xi)\right\|_{p^{\prime}} & \leq C\left\|f-\tau_{\xi} f\right\|_{W_{p^{\prime}}^{2 \widetilde{s}-1-\frac{2}{p}+\rho}}\left\|\chi_{j}^{\varepsilon}\left(\varphi-\varphi_{\delta}\right)\right\|_{W_{p^{\prime}}^{2-\widetilde{s}-\rho}} \\
& \leq K \delta^{\rho+\widetilde{s}-s}\left\|f-\tau_{\xi} f\right\|_{W_{p^{\prime}}^{2 s-1-2 / p}}\|\varphi\|_{W_{p^{\prime}}^{2-s}} \cdot
\end{aligned}
$$

In remains to estimate the function $T_{2}(\xi), \xi \in \mathbb{R}$. To this end we choose $a_{j, \varepsilon} \in \mathbb{R}$ such that supp $\chi_{j}^{\varepsilon} \subset\left[a_{j, \varepsilon}-3 \varepsilon / 2, a_{j, \varepsilon}+3 \varepsilon / 2\right]$ and denote by $F_{\varepsilon}$ the Lipschitz continuous function defined by $F_{\varepsilon}=f$ on $\left[a_{j, \varepsilon}-2 \varepsilon, a_{j, \varepsilon}+2 \varepsilon\right]$ and $F_{\varepsilon}^{\prime}=f^{\prime}\left(x_{j}^{\varepsilon}\right)$ on $\mathbb{R} \backslash\left[a_{j, \varepsilon}-2 \varepsilon, a_{j, \varepsilon}+2 \varepsilon\right]$. Given $\xi \in \mathbb{R}$ with $|\xi| \geq \varepsilon / 2$, it follows from Lemma 2.1 and (70) that

$$
\left\|T_{2}(\xi)\right\|_{p^{\prime}} \leq C\left\|\varphi-\varphi_{\delta}\right\|_{p^{\prime}} \leq C \delta^{2-s}\|\varphi\|_{W_{p^{\prime}}^{2-s}}
$$

If $|\xi|<\varepsilon / 2$, then $\xi+\operatorname{supp} \chi_{j}^{\varepsilon} \subset\left[a_{j, \varepsilon}-2 \varepsilon, a_{j, \varepsilon}+2 \varepsilon\right]$, and, since $f^{\prime} \in \mathrm{C}^{s-1-1 / p}(\mathbb{R})$, Lemma 2.1 and the properties defining $F_{\varepsilon}$ lead to

$$
\begin{aligned}
\left\|T_{2}(\xi)\right\|_{p^{\prime}} & =\left\|\chi_{j}^{\varepsilon} B_{n, m}(f, \ldots, f)\left[f, \ldots, f, F_{\varepsilon}-f^{\prime}\left(x_{j}^{\varepsilon}\right) \operatorname{id}_{\mathbb{R}}, \chi_{j}^{\varepsilon}\left(\varphi-\varphi_{\delta}\right)-\tau_{\xi}\left(\chi_{j}^{\varepsilon}\left(\varphi-\varphi_{\delta}\right)\right)\right]\right\|_{p^{\prime}} \\
& \leq C\left\|f^{\prime}-f^{\prime}\left(x_{j}^{\varepsilon}\right)\right\|_{L_{\infty}\left(\left(a_{j, \varepsilon}-2 \varepsilon, a_{j, \varepsilon}+2 \varepsilon\right)\right)}\left\|\chi_{j}^{\varepsilon}\left(\varphi-\varphi_{\delta}\right)-\tau_{\xi}\left(\chi_{j}^{\varepsilon}\left(\varphi-\varphi_{\delta}\right)\right)\right\|_{p^{\prime}} \\
& \leq \frac{v}{24}\left\|\chi_{j}^{\varepsilon}\left(\varphi-\varphi_{\delta}\right)-\tau_{\xi}\left(\chi_{j}^{\varepsilon}\left(\varphi-\varphi_{\delta}\right)\right)\right\|_{p^{\prime}}
\end{aligned}
$$

provided that $\varepsilon$ is sufficiently small.

Combining (76)-(79), we conclude that if $\varepsilon$ is sufficiently small, then

$$
\begin{aligned}
{\left[T_{j, \varepsilon}^{1}[\varphi]\right]_{W_{p^{\prime}}^{2-s}} } & \leq 3\left(\sum_{j=1}^{3} \int_{\mathbb{R}} \frac{\left\|T_{i}(\xi)\right\|_{p^{\prime}}^{p^{\prime}}}{|\xi|^{1+(2-s) p^{\prime}}} d \xi\right)^{1 / p^{\prime}} \\
& \leq K \delta^{\rho+\widetilde{s}-s}\|\varphi\|_{W_{p^{\prime}}^{2-s}}\left(1+\left\|\chi_{j}^{\varepsilon}\right\|_{W_{p^{\prime}}^{3-s}}+\|f\|_{W_{p}^{s}}\right)+\frac{v}{8}\left\|\chi_{j}^{\varepsilon}\left(\varphi-\varphi_{\delta}\right)\right\|_{W_{p^{\prime}}^{2-s}} .
\end{aligned}
$$

Invoking (4) and Lemma 4.3 (i), the estimates (75) and (80) lead us to

$$
\left\|T_{j, \varepsilon}^{1}[\varphi]\right\|_{W_{p^{\prime}}^{2-s}} \leq \frac{v}{2}\|\varphi\|_{W_{p^{\prime}}^{2-s}}+K \delta^{\rho+\widetilde{s}-s}\|\varphi\|_{W_{p^{\prime}}^{2-s}}
$$

We now chose $\delta=\delta(\varepsilon) \in(0,1]$ sufficiently small to ensure that $K \delta^{\rho+\widetilde{s}-s} \leq v / 2$. This choice together with (74) shows that the estimates in (73) hold true and the proof is complete.

The next lemma is the second main result of this section and describes how to freeze the kernels at infinity. 
Lemma 4.5 Let $n, m \in \mathbb{N}, p \in(1,2], s \in(1+1 / p, 2), \rho \in(0,(s-1-1 / p) / 2)$, $\nu \in(0, \infty)$, and $f \in W_{p}^{s}(\mathbb{R})$ be given. For sufficiently small $\varepsilon \in(0,1)$ there exists a constant $K$ that depends on $\varepsilon, n, m, s, p, \rho$, and $\|f\|_{W_{p}^{s}}$ such that

$$
\left\|\pi_{N}^{\varepsilon} B_{0, m}^{0}(f)[\bar{\omega}]-B_{0,0}\left[\pi_{N}^{\varepsilon} \bar{\omega}\right]\right\|_{W_{p}^{s-2}} \leq v\left\|\pi_{N}^{\varepsilon} \bar{\omega}\right\|_{W_{p}^{s-2}}+K\|\bar{\omega}\|_{W_{p}^{s-2-\rho}}
$$

and

$$
\left\|\pi_{N}^{\varepsilon} B_{n, m}^{0}(f)[\bar{\omega}]\right\|_{W_{p}^{s-2}} \leq v\left\|\pi_{N}^{\varepsilon} \bar{\omega}\right\|_{W_{p}^{s-2}}+K\|\bar{\omega}\|_{W_{p}^{s-2-\rho}, \quad n \geq 1}
$$

for all $\bar{\omega} \in W_{p}^{s-2}(\mathbb{R})$.

Proof Similarly as in the proof of Lemma 4.2 we write

$$
\pi_{N}^{\varepsilon} B_{0, m}^{0}(f)[\bar{\omega}]-B_{0,0}\left[\pi_{N}^{\varepsilon} \bar{\omega}\right]=T_{a}+T_{b},
$$

where

$$
\begin{aligned}
T_{a} & :=\left(\chi_{N}^{\varepsilon} B_{0,0}\left[\pi_{N}^{\varepsilon} \bar{\omega}\right]-B_{0,0}\left[\chi_{N}^{\varepsilon}\left(\pi_{N}^{\varepsilon} \bar{\omega}\right)\right]\right)-\chi_{N}^{\varepsilon}\left(B_{0, m}^{0}(f)\left[\pi_{N}^{\varepsilon} \bar{\omega}\right]-\pi_{N}^{\varepsilon} B_{0, m}^{0}(f)[\bar{\omega}]\right), \\
T_{b} & :=\chi_{N}^{\varepsilon}\left(B_{0, m}^{0}(f)\left[\pi_{N}^{\varepsilon} \bar{\omega}\right]-B_{0,0}\left[\pi_{N}^{\varepsilon} \bar{\omega}\right]\right) .
\end{aligned}
$$

Since $\pi_{j}^{\varepsilon}$ is a pointwise multiplier for $W_{p}^{s-2-\rho}(\mathbb{R})$ and $\chi_{j}^{\varepsilon}$ is a pointwise multiplier for $W_{p}^{s-2}(\mathbb{R})$, we get in view of Lemma 4.1 that

$$
\left\|T_{a}\right\|_{W_{p}^{s-2}} \leq K\|\bar{\omega}\|_{W_{p}^{s-2-\rho}}
$$

With respect to $T_{b}$ we infer from (14) that

$$
T_{b}=-\chi_{N}^{\varepsilon} \sum_{i=1}^{m} B_{2, i}^{0}(f)\left[\pi_{N}^{\varepsilon} \bar{\omega}\right] .
$$

If $\varepsilon$ sufficiently small, it follows from Lemma 4.6 that

$$
\left\|T_{b}\right\|_{W_{p}^{s-2}} \leq v\left\|\pi_{N}^{\varepsilon} \bar{\omega}\right\|_{W_{p}^{s-2}}+K\|\bar{\omega}\|_{W_{p}^{s-2-\rho}} .
$$

The claim (81) follows now from (83) and (84). Finally, the assertion (82) is obtained by arguing as in the proof of (81).

Lemma 4.6 below provides the crucial estimate in the proof of Lemma 4.5.

Lemma 4.6 Let $n, m \in \mathbb{N}, \quad n \geq 1, \quad p \in(1,2], \quad 1+1 / p<\tilde{s}<s<2, \quad v \in(0, \infty)$, and $f \in W_{p}^{s}(\mathbb{R})$ be given. For sufficiently small $\varepsilon \in(0,1)$, there exists a positive constant $K=K\left(\varepsilon, n, m, s, \widetilde{s},\|f\|_{W_{p}^{s}}\right)$ such that

$$
\left\|\chi_{N}^{\varepsilon} B_{n, m}^{0}(f)\left[\pi_{N}^{\varepsilon} \bar{\omega}\right]\right\|_{W_{p}^{s-2}} \leq v\left\|\pi_{N}^{\varepsilon} \bar{\omega}\right\|_{W_{p}^{s-2}}+K\|\bar{\omega}\|_{W_{p}^{\widetilde{s}-2}}
$$


for all $\bar{\omega} \in W_{p}^{s-2}(\mathbb{R})$.

Proof Given $\varphi, \bar{\omega} \in \mathrm{C}_{0}^{\infty}(\mathbb{R})$, it follows by arguing as in the proof of Lemma 4.4 that

$$
\left\langle\chi_{N}^{\varepsilon} B_{n, m}^{0}(f)\left[\pi_{N}^{\varepsilon} \bar{\omega}\right] \mid \varphi\right\rangle_{W_{p}^{s-2(\mathbb{R}) \times W_{p^{\prime}}^{2-s}(\mathbb{R})}}=-\left\langle\pi_{N}^{\varepsilon} \bar{\omega} \mid \chi_{N}^{\varepsilon} B_{n, m}^{0}(f)\left[\chi_{N}^{\varepsilon} \varphi\right]\right\rangle_{W_{p}^{s-2}(\mathbb{R}) \times W_{p^{\prime}}^{2-s}(\mathbb{R})} .
$$

We write $\chi_{N}^{\varepsilon} B_{n, m}^{0}(f)\left[\chi_{N}^{\varepsilon} \varphi\right]=T_{\varepsilon}^{1}[\varphi]+T_{\varepsilon}^{2}[\varphi]$ where, for sufficiently small $\varepsilon$, we have

$$
\left\|T_{\varepsilon}^{1}[\varphi]\right\|_{W_{p^{\prime}}^{2-s}} \leq v\|\varphi\|_{W_{p^{\prime}}^{2-s}} \quad \text { and } \quad\left\|T_{\varepsilon}^{2}[\varphi]\right\|_{W_{p^{\prime}}^{2-s}} \leq K\|\varphi\|_{W_{p^{\prime}}^{2-s}}
$$

for all $\varphi \in W_{p^{\prime}}^{2-s}(\mathbb{R})$. The estimates in (86) together with the previous identity imply

$$
\begin{aligned}
& \left|\left\langle\chi_{N}^{\varepsilon} B_{n, m}^{0}(f)\left[\pi_{N}^{\varepsilon} \bar{\omega}\right] \mid \varphi\right\rangle_{W_{p}^{s-2}(\mathbb{R}) \times W_{p^{\prime}}^{2-s}(\mathbb{R})}\right| \\
& \leq\left\|\pi_{N}^{\varepsilon} \bar{\omega}\right\|_{W_{p}^{s-2}}\left\|T_{\varepsilon}^{1}[\varphi]\right\|_{W_{p^{\prime}}^{2-s}}+\left\|\pi_{N}^{\varepsilon} \bar{\omega}\right\|_{W_{p}^{\widetilde{s}-2}}\left\|T_{\varepsilon}^{2}[\varphi]\right\|_{W_{p^{\prime}}^{2-s}} \\
& \leq\left(v\left\|\pi_{N}^{\varepsilon} \bar{\omega}\right\|_{W_{p}^{s-2}}+K\|\bar{\omega}\|_{W_{p}^{s-2}}\right)\|\varphi\|_{W_{p^{\prime}}^{2-s}},
\end{aligned}
$$

and (85) follows.

Let again $\left\{\eta_{\delta}\right\}_{\delta>0}$ be a mollifier and set

$$
T_{\varepsilon}^{1}[\varphi]=\chi_{N}^{\varepsilon} B_{n, m}^{0}(f)\left[\chi_{N}^{\varepsilon}\left(\varphi-\varphi_{\delta}\right)\right] \quad \text { and } \quad T_{\varepsilon}^{2}[\varphi]=\chi_{N}^{\varepsilon} B_{n, m}^{0}(f)\left[\chi_{N}^{\varepsilon} \varphi_{\delta}\right],
$$

where $\varphi_{\delta}:=\varphi * \eta_{\delta}$ and with $\delta=\delta(\varepsilon) \in(0,1]$ which we fix later on. Taking advantage of Lemma 2.4 (with $r=2-\widetilde{s} \in(0,1-1 / p)$ ) and of the fact that $\chi_{N}^{\varepsilon} \varphi_{\delta} \in W_{p^{\prime}}^{2-\widetilde{s}}(\mathbb{R})$, we conclude that $T_{\varepsilon}^{2}[\varphi] \in W_{p^{\prime}}^{2-\widetilde{s}}(\mathbb{R})$ and together with (69) we obtain the estimate

$$
\left\|T_{\varepsilon}^{2}[\varphi]\right\|_{W_{p^{\prime}}^{2-\widetilde{s}}} \leq K\left\|\chi_{N}^{\varepsilon} \varphi_{\delta}\right\|_{W_{p^{\prime}}^{2-\widetilde{s}}} \leq K \delta^{\widetilde{s}-s}\|\varphi\|_{W_{p^{\prime}}^{2-s}}
$$

We now estimate $T_{\varepsilon}^{1}[\varphi]$. Combining Lemma 2.1 and (70), we have

$$
\left\|T_{\varepsilon}^{1}[\varphi]\right\|_{p^{\prime}} \leq C\left\|\chi_{N}^{\varepsilon}\left(\varphi_{\delta}-\varphi\right)\right\|_{p^{\prime}} \leq C\left\|\varphi-\varphi_{\delta}\right\|_{p^{\prime}} \leq C \delta^{2-s}\|\varphi\|_{W_{p^{\prime}}^{2-s}},
$$

and it remains to consider the seminorm $\left[T_{\varepsilon}^{1}[\varphi]\right]_{W_{p^{\prime}}^{2-s .}}$ Using (14), we get

$$
T_{\varepsilon}^{1}[\varphi]-\tau_{\xi} T_{\varepsilon}^{1}[\varphi]=T_{1}(\xi)+T_{2}(\xi)+T_{3}(\xi), \quad \xi \in \mathbb{R},
$$

where 


$$
\begin{aligned}
T_{1}(\xi) & :=\left(\chi_{N}^{\varepsilon}-\tau_{\xi} \chi_{N}^{\varepsilon}\right) \tau_{\xi}\left(B_{n, m}^{0}(f)\left[\chi_{N}^{\varepsilon}\left(\varphi-\varphi_{\delta}\right)\right]\right), \\
T_{2}(\xi) & :=\chi_{N}^{\varepsilon} B_{n, m}^{0}(f)\left[\chi_{N}^{\varepsilon}\left(\varphi-\varphi_{\delta}\right)-\tau_{\xi}\left(\chi_{N}^{\varepsilon}\left(\varphi-\varphi_{\delta}\right)\right)\right], \\
T_{3}(\xi) & :=\chi_{N}^{\varepsilon} \sum_{j=1}^{n} B_{n, m}(f, \ldots, f)[\underbrace{\tau_{\xi} f, \ldots, \tau_{\xi}}_{(j-1)-\text { times }}, f-\tau_{\xi} f, f, \ldots, f, \tau_{\xi}\left(\chi_{N}^{\varepsilon}\left(\varphi-\varphi_{\delta}\right)\right)] \\
+ & \chi_{N}^{\varepsilon} \sum_{j=1}^{m} B_{n+2, m+1}^{j}\left[\tau_{\xi} f, \ldots, \tau_{\xi} f, \tau_{\xi} f+f, \tau_{\xi} f-f, \tau_{\xi}\left(\chi_{N}^{\varepsilon}\left(\varphi-\varphi_{\delta}\right)\right)\right],
\end{aligned}
$$

with $B_{n+2, m+1}^{j}$ as defined in the proof of Lemma 4.4. Arguing as in Lemma 4.4, we obtain

$$
\left\|T_{1}(\xi)\right\|_{p^{\prime}} \leq C \delta^{2-s}\left\|\chi_{N}^{\varepsilon}-\tau_{\xi} \chi_{N}^{\varepsilon}\right\|_{W_{p^{\prime}}^{1}}\|\varphi\|_{W_{p^{\prime}}^{2-s}}
$$

and, for some fixed $\rho \in(s-\widetilde{s}, \min \{2-\widetilde{s}, 2(s-\widetilde{s})\})$,

$$
\left\|T_{3}(\xi)\right\|_{p^{\prime}} \leq K \delta^{\rho+\widetilde{s}-s}\left\|f-\tau_{\xi} f\right\|_{W_{p^{\prime}}^{2 s-1-2 / p}}\|\varphi\|_{W_{p^{\prime}}^{2-s}} .
$$

In order to estimate $T_{2}(\xi)$, let $F_{\varepsilon}$ denote the Lipschitz continuous function equal to $f$ on the set $\{|x| \geq 1 / \varepsilon-2 \varepsilon\}$ and which is linear in the interval $\{|x| \leq 1 / \varepsilon-2 \varepsilon\}$. If $|\xi| \geq \varepsilon$, Lemma 2.1 and (70) yield

$$
\left\|T_{2}(\xi)\right\|_{p^{\prime}} \leq C\left\|\varphi-\varphi_{\delta}\right\|_{p^{\prime}} \leq C \delta^{2-s}\|\varphi\|_{W_{p^{\prime}}^{2-s}}
$$

If $|\xi|<\varepsilon$, we note that $\xi+\operatorname{supp} \chi_{N}^{\varepsilon} \subset\{|x| \geq 1 / \varepsilon-2 \varepsilon\}$. Lemma 2.1, the definition of $F_{\varepsilon}$, and the observation that $\left\|F_{\varepsilon}^{\prime}\right\|_{\infty} \leq 2 \varepsilon\|f\|_{\infty}+\left\|f^{\prime}\right\|_{L_{\infty}(\{|x| \geq 1 / \varepsilon-2 \varepsilon\})}$ (which holds if $\varepsilon$ is sufficiently small), then lead to

$$
\begin{aligned}
\left\|T_{2}(\xi)\right\|_{p^{\prime}} & =\left\|\chi_{N}^{\varepsilon} B_{n, m}(f, \ldots, f)\left[f, \ldots, f, F_{\varepsilon}, \chi_{N}^{\varepsilon}\left(\varphi-\varphi_{\delta}\right)-\tau_{\xi}\left(\chi_{N}^{\varepsilon}\left(\varphi-\varphi_{\delta}\right)\right)\right]\right\|_{p^{\prime}} \\
& \leq C\left\|F_{\varepsilon}^{\prime}\right\|_{\infty}\left\|\chi_{N}^{\varepsilon}\left(\varphi-\varphi_{\delta}\right)-\tau_{\xi}\left(\chi_{N}^{\varepsilon}\left(\varphi-\varphi_{\delta}\right)\right)\right\|_{p^{\prime}} \\
& \leq \frac{v}{24}\left\|\chi_{N}^{\varepsilon}\left(\varphi-\varphi_{\delta}\right)-\tau_{\xi}\left(\chi_{N}^{\varepsilon}\left(\varphi-\varphi_{\delta}\right)\right)\right\|_{p^{\prime}}
\end{aligned}
$$

provided that $\varepsilon$ is sufficiently small. To obtain the last inequality we have taken advantage of the fact that $f^{\prime} \in W_{p}^{s-1}(\mathbb{R})$ vanishes at infinity.

From (89) to (92), we conclude, for $\varepsilon$ sufficiently small, that

$$
\left[T_{\varepsilon}^{1}[\varphi]\right]_{W_{p^{\prime}}^{2-s}} \leq K \delta^{\rho+\widetilde{s}-s}\|\varphi\|_{W_{p^{\prime}}^{2-s}}\left(1+\left\|1-\chi_{N}^{\varepsilon}\right\|_{W_{p^{\prime}}^{3-s}}+\|f\|_{W_{p}^{s}}\right)+\frac{v}{8}\left\|\chi_{N}^{\varepsilon}\left(\varphi-\varphi_{\delta}\right)\right\|_{W_{p^{\prime}}^{2-s}}
$$

Combining (4), Lemma 4.3 (i), (88), and (93) we get 


$$
\left\|T_{\varepsilon}^{1}[\varphi]\right\|_{W_{p^{\prime}}^{2-s}} \leq \frac{v}{2}\|\varphi\|_{W_{p^{\prime}}^{2-s}}+K \delta^{\rho+\widetilde{s}-s}\|\varphi\|_{W_{p^{\prime}}^{2-s}}
$$

Choosing now $\delta=\delta(\varepsilon)$ sufficiently small to ensure that $K \delta^{\rho+\widetilde{s}-s} \leq v / 2$, we obtain, together with (87), the desired estimates (86) and the proof is complete.

\section{Estimates for some pointwise multipliers}

In this appendix we present the proofs of the estimates (6)-(7). The estimate (7) is used in the proof of Theorem 3.3 and (6) is an important argument when establishing (7).

Proof of (6) Since $W_{p}^{r}(\mathbb{R}) \hookrightarrow \mathrm{C}(\mathbb{R})$, we have

$$
\|g h\|_{p^{\prime}} \leq\|g\|_{\infty}\|h\|_{p^{\prime}}
$$

and it remains to estimate the term

$$
[g h]_{W_{p^{\prime}}}^{p^{\prime}}=\int_{\mathbb{R}} \frac{\left\|g h-\tau_{\xi}(g h)\right\|_{p^{\prime}}^{p^{\prime}}}{|\xi|^{1+(1-r) p^{\prime}}} d \xi \leq 2^{p^{\prime}}\left(\|g\|_{\infty}^{p^{\prime}} \int_{\mathbb{R}} \frac{\left\|h-\tau_{\xi} h\right\|_{p^{\prime}}^{p^{\prime}}}{|\xi|^{1+(1-r) p^{\prime}}} d \xi+\int_{\mathbb{R}} \frac{\left\|\left(g-\tau_{\xi} g\right) \tau_{\xi} h\right\|_{p^{\prime}}^{p^{\prime}}}{|\xi|^{1+(1-r) p^{\prime}}} d \xi\right) .
$$

According to [5, Theorem 4.1], the multiplication

$$
[(g, h) \mapsto g h]: W_{p^{\prime}}^{2(r-1 / p)}(\mathbb{R}) \times W_{p^{\prime}}^{1-r-\rho}(\mathbb{R}) \rightarrow L_{p^{\prime}}(\mathbb{R})
$$

is continuous, hence

$$
[g h]_{W_{p^{\prime}}^{1-r}}^{p^{\prime}} \leq 2^{p^{\prime}}\|g\|_{\infty}^{p^{\prime}}\|h\|_{W_{p^{\prime}}^{1-r}}^{p^{\prime}}+C\|h\|_{W_{p^{\prime}}^{1-r-\rho}}^{p^{\prime}} \int_{\mathbb{R}} \frac{\left\|g-\tau_{\xi} g\right\|_{W_{p^{\prime}}^{2(r-1 / p)}}^{p^{\prime}}}{|\xi|^{1+(1-r) p^{\prime}}} d \xi .
$$

Lemma 2.3 (with $t^{\prime}=2(r-1 / p)$ and $t=r+1-2 / p$ ) yields

$$
\int_{\mathbb{R}} \frac{\left\|g-\tau_{\xi} g\right\|_{W_{p^{\prime}}^{2(r-1 / p)}}^{p^{\prime}}}{|\xi|^{1+(1-r) p^{\prime}}} d \xi \leq\|g\|_{W_{p^{\prime}}^{r+1-2 / p}}^{p^{\prime}} \leq C\|g\|_{W_{p}^{r}}^{p^{\prime}} .
$$

We thus conclude that

$$
[g h]_{W_{p^{\prime}}^{1-r}}^{p^{\prime}} \leq 2^{p^{\prime}}\|g\|_{\infty}^{p^{\prime}}\|h\|_{W_{p^{\prime}}^{1-r}}^{p^{\prime}}+C\|g\|_{W_{p}^{r}}^{p^{\prime}}\|h\|_{W_{p^{\prime}}^{1-r-\rho}}^{p^{\prime}},
$$

and (94) together with (95) lead to the desired claim.

We are now in a position to prove (7). 
Proof of (7) Given $h, \psi \in \mathrm{C}_{0}^{\infty}(\mathbb{R})$, we have

$$
\langle\varphi h \mid \psi\rangle_{W_{p}^{r-1}(\mathbb{R}) \times W_{p^{\prime}}^{1-r}(\mathbb{R})}=\langle h \mid \varphi \psi\rangle_{W_{p}^{r-1}(\mathbb{R}) \times W_{p^{\prime}}^{1-r}(\mathbb{R})}=\left\langle h \mid T_{1}[\psi]+T_{2}[\psi]\right\rangle_{W_{p}^{r-1}(\mathbb{R}) \times W_{p^{\prime}}^{1-r}(\mathbb{R})},
$$

where $T_{1}[\psi] \in W_{p^{\prime}}^{1-r}(\mathbb{R})$ and $T_{2}[\psi] \in W_{p^{\prime}}^{1-r+\rho}(\mathbb{R})$ are defined in (97) and satisfy

$$
\left\|T_{1}[\psi]\right\|_{W_{p^{\prime}}^{1-r}} \leq 5\|\varphi\|_{\infty}\|\psi\|_{W_{p^{\prime}}^{1-r}} \quad \text { and } \quad\left\|T_{2}[\psi]\right\|_{W_{p^{\prime}}^{1-r+\rho}} \leq C \frac{\|\varphi\|_{W_{p}^{r}}^{1+2 r / \rho}}{\|\varphi\|_{\infty}^{2 r / \rho}}\|\psi\|_{W_{p^{\prime}}^{1-r}}^{2 .}
$$

Having established (96), we get

$$
\begin{aligned}
|\langle\varphi h \mid \psi\rangle|_{W_{p}^{r-1}(\mathbb{R}) \times W_{p^{\prime}}^{1-r}(\mathbb{R})} & \leq\|h\|_{W_{p}^{r-1}}\left\|T_{1}[\psi]\right\|_{W_{p^{\prime}}^{1-r}}+\|h\|_{W_{p}^{r-1-\rho}}\left\|T_{2}[\psi]\right\|_{W_{p^{\prime}}^{1-r+\rho}} \\
& \leq\left(5\|\varphi\|_{\infty}\|h\|_{W_{p}^{r-1}}+C \frac{\|\varphi\|_{W_{p}^{r}}^{1+2 r / \rho}}{\|\varphi\|_{\infty}^{2 r / \rho}}\|h\|_{W_{p}^{r-1-\rho}}\right)\|\psi\|_{W_{p^{\prime}}^{1-r}},
\end{aligned}
$$

and the estimate (7) follows. The functions in (96) are defined by

$$
T_{1}[\psi]:=\varphi\left(\psi-\psi_{\delta}\right) \quad \text { and } \quad T_{2}[\psi]:=\varphi \psi_{\delta},
$$

where $\left\{\eta_{\delta}\right\}_{\delta>0}$ is a mollifier, $\psi_{\delta}=\psi * \eta_{\delta}$, and $\delta \in(0,1]$ is chosen below. Combining (6), Lemma 4.3 (i), and (70), we get

$$
\begin{aligned}
\left\|T_{1}[\psi]\right\|_{W_{p^{\prime}}^{1-r}} & \leq 2\|\varphi\|_{\infty}\left\|\psi-\psi_{\delta}\right\|_{W_{p^{\prime}}^{1-r}}+C\|\varphi\|_{W_{p}^{r}}\left\|\psi-\psi_{\delta}\right\|_{W_{p^{\prime}}^{1-r-\rho}} \\
& \leq\left(4\|\varphi\|_{\infty}+C \delta^{\rho}\|\varphi\|_{W_{p}^{r}}\right)\|\psi\|_{W_{p^{\prime}}^{1-r}} .
\end{aligned}
$$

After eventually choosing $C$ to be larger than the norm of the embedding $W_{p}^{r}(\mathbb{R}) \hookrightarrow L_{\infty}(\mathbb{R})$, we set $\delta=\left(\|\varphi\|_{\infty} /\left(C\|\varphi\|_{W_{p}^{r}}\right)\right)^{1 / \rho} \in(0,1]$, and obtain that

$$
\left\|T_{1}[\psi]\right\|_{W_{p^{\prime}}^{1-r}} \leq 5\|\varphi\|_{\infty}\|\psi\|_{W_{p^{\prime}}^{1-r}} .
$$

With respect to $T_{2}[\psi]$, we note that since $\varphi \in W_{p^{\prime}}^{r+1-2 / p}(\mathbb{R})$ and $\psi_{\delta} \in W_{p^{\prime}}^{r+1}(\mathbb{R})$, it holds that $T_{2}[\psi] \in W_{p^{\prime}}^{r+1-2 / p}(\mathbb{R}) \hookrightarrow W_{p^{\prime}}^{1-r+\rho}(\mathbb{R})$, cf. (2), and together with (2) and (69) we get

$$
\left\|T_{2}[\psi]\right\|_{W_{p^{\prime}}^{1-r+\rho}} \leq C\left\|\psi_{\delta}\right\|_{W_{p^{\prime}}^{r+1}}\|\varphi\|_{W_{p^{\prime}}^{r+1-2 / p}} \leq C \delta^{-2 r}\|\varphi\|_{W_{p}^{r}}\|\psi\|_{W_{p^{\prime}}^{1-r}} \leq C \frac{\|\varphi\|_{W_{p}^{r}}^{1+2 r / \rho}}{\|\varphi\|_{\infty}^{2 r / \rho}}\|\psi\|_{W_{p^{\prime}}^{1-r}} .
$$

Hence, both estimates in (96) hold true and the proof is complete.

Acknowledgements The authors were partially supported by the RTG 2339 "Interfaces, Complex Structures, and Singular Limits" of the German Science Foundation (DFG). The support is gratefully acknowledged. 
Funding Open Access funding enabled and organized by Projekt DEAL.

\section{Declarations}

Conflict of interest The authors declare that there is no conflict of interest regarding the publication of this paper.

Ethical approval This article does not contain any studies with human participants or animals performed by the author

Open Access This article is licensed under a Creative Commons Attribution 4.0 International License, which permits use, sharing, adaptation, distribution and reproduction in any medium or format, as long as you give appropriate credit to the original author(s) and the source, provide a link to the Creative Commons licence, and indicate if changes were made. The images or other third party material in this article are included in the article's Creative Commons licence, unless indicated otherwise in a credit line to the material. If material is not included in the article's Creative Commons licence and your intended use is not permitted by statutory regulation or exceeds the permitted use, you will need to obtain permission directly from the copyright holder. To view a copy of this licence, visit http://creativecommons.org/licen ses/by/4.0\%.

\section{References}

1. Abels, H.: Pseudodifferential and Singular Integral Operators. An Introduction with Applications. De Gruyter Graduate Lectures, DeGruyter, Berlin (2012)

2. Abels, H., Matioc, B.-V.: Well-possedness of the Muskat problem in subcritical $L_{p}$-Sobolev spaces, Eur. J. Appl. Math., p. 1-43 (2021), arXiv:2003.07656

3. Amann, H.: Quasilinear parabolic systems under nonlinear boundary conditions. Arch. Ration. Mech. Anal. 92, 153-192 (1986)

4. Amann, H.: Dynamic theory of quasilinear parabolic equations. I. Abstract evolution equations. Nonlinear Anal. 12, 895-919 (1988)

5. Amann, H.: Multiplication in Sobolev and Besov spaces. In: Nonlinear analysis, Sc. Norm. Super. di Pisa Quaderni, Scuola Norm. Sup., Pisa, pp. 27-50 (1991)

6. Amann, H.: Nonhomogeneous linear and quasilinear elliptic and parabolic boundary value problems. In: Schmeisser, H.-J., et al. (ed.) Function Spaces, Diferential Operators and Nonlinear Analysis. Survey Articles and Communications of the International Conference Held in Friedrichsroda, Germany, September 20-26, 1992, vol. 133 of Teubner-Texte Math. B. G. Teubner Verlagsgesellschaft, Stuttgart, pp. 9-126 (1993)

7. Amann, H.: Amann, Linear and Quasilinear Parabolic Problems. Vol. I, vol. 89 of Monographs in Mathematics. Birkhäuser Boston, Inc., Boston (1995).. ((Abstract linear theory))

8. Ambrose, D.M.: The zero surface tension limit of two-dimensional interfacial Darcy flow. J. Math. Fluid Mech. 16, 105-143 (2014)

9. Angenent, S.B.: Nonlinear analytic semiflows. Proc. R. Soc. Edinb. Sect. A 115, 91-107 (1990)

10. Bazaliy, B.V., Vasylyeva, N.: The Muskat problem with surface tension and a nonregular initial interface. Nonlinear Anal. 74, 6074-6096 (2011)

11. Constantin, A., Escher, J.: Analyticity of periodic traveling free surface water waves with vorticity. Ann. Math. 173, 559-568 (2011)

12. Constantin, P., Gancedo, F., Shvydkoy, R., Vicol, V.: Global regularity for 2D Muskat equations with finite slope. Ann. Inst. H. Poincaré Anal. Non Linéaire 34, 1041-1074 (2017)

13. Ehrnström, M., Escher, J., Matioc, B.-V.: Steady-state fingering patterns for a periodic Muskat problem. Methods Appl. Anal. 20, 33-46 (2013)

14. Escher, J., Matioc, A.-V., Matioc, B.-V.: A generalized Rayleigh-Taylor condition for the Muskat problem. Nonlinearity 25, 73-92 (2012) 
15. Escher, J., Matioc, A.-V., Matioc, B.-V.: Modelling and analysis of the Muskat problem for thin fluid layers. J. Math. Fluid Mech. 14, 267-277 (2012)

16. Escher, J., Matioc, B.-V.: On the parabolicity of the Muskat problem: well-posedness, fingering, and stability results. Z. Anal. Anwend. 30, 193-218 (2011)

17. Escher, J., Matioc, B.-V., Walker, C.: The domain of parabolicity for the Muskat problem. Indiana Univ. Math. J. 67, 679-737 (2018)

18. Escher, J., Simonett, G.: Analyticity of the interface in a free boundary problem. Math. Ann. 305, 439-459 (1996)

19. Escher, J., Simonett, G.: Classical solutions for Hele-Shaw models with surface tension. Adv. Differ. Equ. 2, 619-642 (1997)

20. Friedman, A., Tao, Y.: Nonlinear stability of the Muskat problem with capillary pressure at the free boundary. Nonlinear Anal. 53, 45-80 (2003)

21. Gancedo, F., Granero-Belinchón, R., Scrobogna, S.: Surface tension stabilization of the Rayleigh-Taylor instability for a fluid layer in a porous medium. Ann. Inst. Henri Poincaré, Anal. Non Linéaire 37(6), 1299-1343 (2020)

22. Granero-Belinchón, R., Scrobogna, S.: Asymptotic models for free boundary flow in porous media. Phys. D 392, 1-16 (2019)

23. Hong, J., Tao, Y., Yi, F.: Muskat problem with surface tension. J. Partial Differ. Equ. 10, 213231 (1997)

24. Matioc, B.-V.: Viscous displacement in porous media: the Muskat problem in 2D. Trans. Am. Math. Soc. 370, 7511-7556 (2018)

25. Matioc, B.-V.: The Muskat problem in two dimensions: equivalence of formulations, well-posedness, and regularity results. Anal. PDE 12, 281-332 (2019)

26. Matioc, B.-V.: Well-posedness and stability results for some periodic Muskat problems. J. Math. Fluid Mech. 22, 31 (2020). ((45))

27. Matioc, B.-V., Prokert, G.: Two-phase Stokes flow by capillarity in full $2 \mathrm{~d}$ space: an approach via hydrodynamic potentials. Proc. R. Soc. Edinb. Sect. A, p. 1-31 (2020)

28. Matioc, B.-V., Walker, C.: On the principle of linearized stability in interpolation spaces for quasilinear evolution equations. Monatsh. Math. 191, 615-634 (2020)

29. Muskat, M.: Two fluid systems in porous media. The encroachment of water into an oil sand. Physics 5, 250-264 (1934)

30. Nguyen, H.Q.: On well-posedness of the Muskat problem with surface tension. Adv. Math. 374, 107344 (2020)

31. Nguyen, H.Q. Flynn, P.T.: The vanishing surface tension limit of the Muskat problem, (2020). arXiv:2020.10473

32. Prüss, J., Shao, Y., Simonett, G.: On the regularity of the interface of a thermodynamically consistent two-phase Stefan problem with surface tension. Interfaces Free Bound. 17, 555-600 (2015)

33. Prüss, J., Simonett, G.: Moving Interfaces and Quasilinear Parabolic Evolution Equations. Monographs in Mathematics, vol. 105. Birkhäuser/Springer, Cham (2016)

34. Prüss, J., Simonett, G.: The Verigin problem with and without phase transition. Interfaces Free Bound. 20, 107-128 (2018)

35. Prüss, J., Simonett, G., Wilke, M.: The Rayleigh-Taylor instability for the Verigin problem with and without phase transition. NoDEA Nonlinear Differ. Equ. Appl. 26, 18 (2019). ((35))

36. Prüss, J., Simonett, G.: On the Muskat flow. Evol. Equ. Control Theory 5, 631-645 (2016)

37. Scrobogna, S.: Well-posedness of an asymptotic model for free boundary Darcy flow in porous media in the critical Sobolev space. Nonlinear Anal. Real World Appl. 60, Article ID 103308 (2021)

38. Tao, Y.: Classical solution of Verigin problem with surface tension. Chin. Ann. Math. Ser. B 18, 393-404 (1997)

39. Tofts, S.: On the existence of solutions to the Muskat problem with surface tension. J. Math. Fluid Mech. 19, 581-611 (2017)

40. Triebel, H.: Theory of function spaces. In: Mathematik und ihre Anwendungen in Physik und Technik [Mathematics and its Applications in Physics and Technology], vol. 38. Akademische Verlagsgesellschaft Geest \& Portig K.-G.284 p., 20 figs. DDR M 69.00, Leipzig (1983)

Publisher's Note Springer Nature remains neutral with regard to jurisdictional claims in published maps and institutional affiliations. 